1 Large amplitude motions of a submerged circular cylinder in water with an ice cover Z.F. Li a , Y.Y. Shi ${ }^{\text {b }}$, and G.X. Wu ${ }^{\text {b,c,* }}$ a School of Naval Architecture and Ocean Engineering, Jiangsu University of Science and 5 Technology, Zhenjiang 212003, China

$6{ }^{b}$ College of Shipbuilding Engineering, Harbin Engineering University, Harbin 150001, China

$7 \quad{ }^{\mathrm{c}}$ Department of Mechanical Engineering, University College London, Torrington Place, London 8 WC1E 7JE, UK

\title{
9 Abstract
}

The hydrodynamic problem of a circular cylinder undergoing large amplitude oscillations in water covered by an ice sheet is investigated. The ice sheet is modelled by a thin elastic sheet and uniform physical properties are assumed. The fluid is assumed to be inviscid, incompressible and homogeneous, and the depth to be infinite. The boundary condition on the ice sheet is linearized and satisfied on its mean position, while the fully nonlinear boundary condition is imposed on the instantaneous position of the body surface. The velocity potential is formulated by the multipole expansion method in the polar coordinate system with its origin fixed at the centre of the cylinder. Detailed results through the hydrodynamic force and deformation of the ice sheet are provided. The effects of the ice sheet properties, and motion amplitude and frequency are investigated.

\section{Keywords}

Ice sheet; circular cylinder; large amplitude oscillation; multipole expansion.

\section{Introduction}

Water wave/structure interaction has always been a major interest in fluid mechanics. This is primarily because of the importance of the ocean. It provides a major means for trade, as well as is a rich source of food and resource. Its wave, current and wind can also provide clean and renewable energy. Therefore, there has been extensive research on this topic. However, the most work focuses on a body in open seas, in which the water surface is in direct contact with air, and the surface is commonly called free surface.

A closely related problem is when the water surface is covered by an ice sheet, which can be typically seen in the Arctic or Antarctic regions. There has also been a large number of work on ice sheet/wave interaction. Review of early work was given by Squire, et al. [1] and more recent

\footnotetext{
${ }^{*}$ Corresponding author. Permanent address: Department of Mechanical Engineering, University College London, Torrington Place, London WCIE 7JE, UK. Tel.: +44 207679 3870; fax: +44 2073880180.

E-mail address: g.wu@ucl.ac.uk (G.X.Wu)
} 
one was given by Squire [2]. Much of the work reviewed is about wave interaction with ice sheet. It is quite often that the ice cover on the water surface is treated as an infinite or semi-infinite elastic sheet or plate. Fox and Squire [3] considered a two dimensional problem of wave from open water propagating into ice sheet. This method was extended later to consider the oblique incidence problem [4]. Sahoo, et al. [5] considered similar problems using an inner product with orthogonality in the eigenfunction expansions method. Eatock Taylor and Ohkusu [6] investigated the flexural vibrations of a uniform elastic plate using the Green function. Other work includes those by Balmforth and Craster [7], Tkacheva [8], Tkacheva [9] and Chung and Fox [10]. More recently, Sturova and Korobkin [11] solved the problem of a floating elastic plate under periodic pressure.

There have also been strong interests in wave/ice/body interaction, especially more recently, as a new shipping through Arctic becomes more feasible in coming decades. Das and Mandal [12] studied the oblique wave scattering by a circular cylinder submerged beneath an ice-cover through the 2-D multipole expansion method. Sturova [13] considered the radiation problems of a horizontal cylinder submerged in fluid covered by an ice sheet with semi-infinite length, and the corresponding Green function was constructed using eigenfunction expansions. The method was then extended to the cases of two semi-infinite elastic plates connected by the vertical and flexural rotational springs [14], and the ice floe or polynya [15]. In addition, Savin and Savin [16] and Il'ichev, et al. [17] used a two-dimensional dipole situated under the ice sheet to investigate the generation of the flexural-gravity waves.

In the above work, the fully linearized theory has been used, in which all the conditions are satisfied on the mean position of the boundary and higher order terms are ignored from the condition. This is valid when the motion amplitude is small. Wu [18] considered a problem of a circular cylinder submerged below the free surface. He has argued that when the body motion is large, the boundary condition has to be satisfied on its instantaneous positon. However, when the body is sufficiently deeply submerged, the free surface boundary condition can still be linearized. The solution of such a model subsequently led to some very interesting and new results. Here we shall consider a circular cylinder in a large amplitude motion below an ice sheet. The body surface boundary condition is satisfied on its exact positon while the condition on the ice sheet is linearized. The ice sheet is treated as a thin elastic beam of infinite extent with uniform properties, and the incompressible velocity potential flow theory is adopted in the fluid domain. The polar coordinate system, with its origin fixed at the centre of the circular cylinder, is introduced to describe the instantaneous body surface boundary condition. The velocity potential is written in terms of the multipole expansion [19] with unknown coefficients. Through this the problem is 
transformed into an infinite system of linear equations. Numerical results for the hydrodynamic force and deformation of the ice sheet are provided and discussions are made. The effect of body oscillation amplitude, frequency, as well as ice thickness are investigated. A far field formula for the horizontal steady force is also derived and has been used for verifying the results.

\section{The problem and mathematical equations}

The problem of a submerged horizontal circular cylinder of radius $a$ undergoing sinusoidal oscillation with frequency $\omega$ in fluid with an ice sheet is sketched in Fig. 1. A Cartesian coordinate system $O-x z$ is defined with the $x$-axis along the undisturbed mean water surface, and the $z$-axis pointing vertically upwards. A body-fixed polar coordinate system $(r, \theta)$ is also defined, with its origin fixed at the centre of the cylinder with its mean position located at $(0,-h)$.

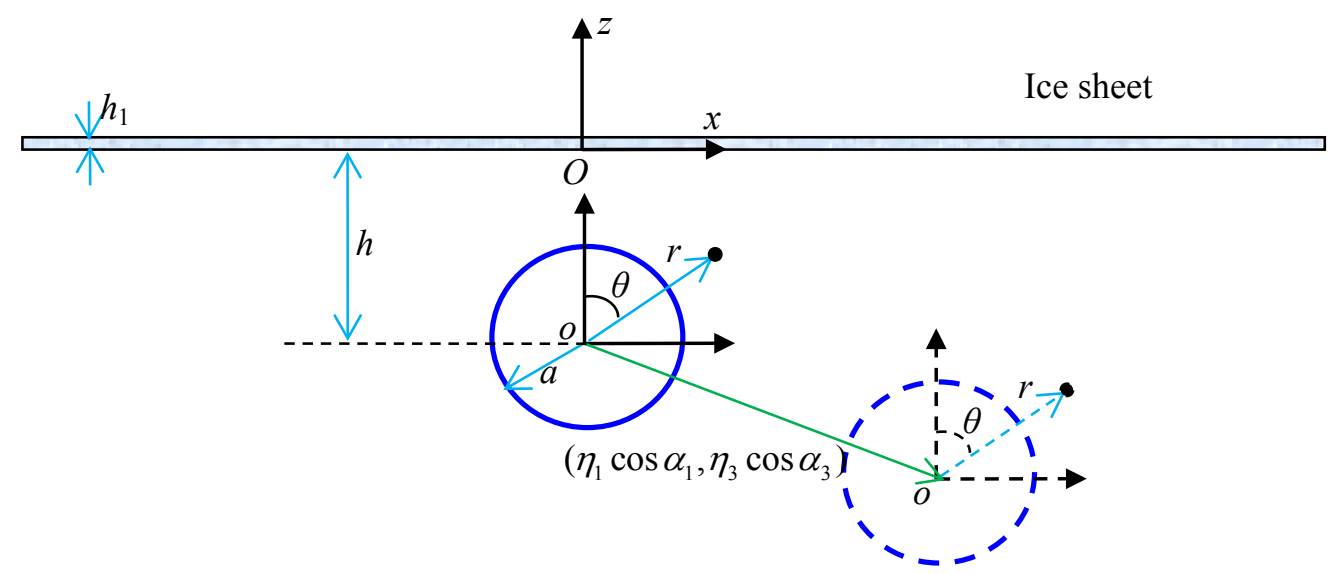

Fig. 1. Definition of the coordinate systems and sketch of the problem.

The relationships between the two coordinate systems can be written as

$$
\begin{gathered}
x=r \sin \theta+\eta_{1} \cos \alpha_{1} \\
z=r \cos \theta-\left(h-\eta_{3} \cos \alpha_{3}\right) \\
\alpha_{j}=\omega t+\gamma_{j}
\end{gathered}
$$

where $\eta_{1}$ and $\eta_{3}$ are the amplitude of horizontal and vertical motions respectively, $\gamma_{1}$ and $\gamma_{3}$ are the corresponding initial phases. The condition $\eta_{3}+a<h$ keeps the cylinder beneath the ice sheet at all time.

The ice sheet with uniform properties is assumed to be in contact with water for all times. The draught of the ice sheet is neglected. The fluid of infinite depth is assumed to be inviscid, incompressible, homogeneous, and the flow is irrotational. Then the velocity potential $\Phi$ can be introduced, which satisfies the Laplace equation in the fluid domain

$$
\nabla^{2} \Phi=0
$$

On the ice sheet $S_{F}$ or $z=0$, the combination of the dynamic and kinematic boundary conditions can be written as [1] 


$$
\left(D \frac{\partial^{4}}{\partial x^{4}}+K \frac{1}{g} \frac{\partial^{2}}{\partial t^{2}}+1\right) \frac{\partial \Phi}{\partial z}+\frac{1}{g} \frac{\partial^{2} \Phi}{\partial t^{2}}=0
$$

where $D=E h_{1}^{3} / 12 \rho g\left(1-v^{2}\right)$ and $K=h_{1} \rho_{1} / \rho$, and $E, v, h_{1}$ and $\rho_{1}$ are respectively the Young's modulus, Poisson's ratio, the thickness and the densities of the ice sheet, and $\rho$ is the density of the fluid.

On the instantaneous body surface $S_{0}$ or $r=a$, the normal velocity of fluid should be equal to that of the body based on the impermeable condition. Thus, we have

$$
\frac{\partial \Phi}{\partial n}=-\omega\left(n_{1} \eta_{1} \sin \alpha_{1}+n_{3} \eta_{3} \sin \alpha_{3}\right)
$$

where $\vec{n}=\left(n_{1}, n_{3}\right)$ is the normal vector pointing into the body, and for the circle above its components along $x$-axis and $z$-axis can be written as $n_{1}=-\sin \theta$ and $n_{3}=-\cos \theta$ respectively. In the far field, i.e. $x \rightarrow \pm \infty$, a radiation condition should be imposed, which requires the radiated waves to propagate outwards.

To solve the above boundary value problem, we may rewrite the total velocity potential in the following form

$$
\Phi=-\omega \eta_{1} \operatorname{Re}\left(\phi_{1} \mathrm{e}^{\mathrm{i} \gamma_{1}}\right)-\omega \eta_{3} \operatorname{Re}\left(\phi_{3} \mathrm{e}^{\mathrm{i} / \gamma_{3}}\right)
$$

$\phi_{j}$ satisfies the same equations as $\Phi$ apart from the body surface boundary condition which can be given as

$$
\begin{aligned}
& \frac{\partial \phi_{1}}{\partial r}=\frac{1}{2}\left(\mathrm{e}^{\mathrm{i}(\omega t+\theta)}-\mathrm{e}^{\mathrm{i}(\omega t-\theta)}\right) \\
& \frac{\partial \phi_{3}}{\partial r}=\frac{\mathrm{i}}{2}\left(\mathrm{e}^{\mathrm{i}(\omega t+\theta)}+\mathrm{e}^{\mathrm{i}(\omega t-\theta)}\right)
\end{aligned}
$$

\section{Method of solution}

In the context of the present problem, the velocity potential can be written in terms of multipole expansion [19]. Each term in the series satisfies the Laplace equation and boundary conditions apart from that on the cylinder surface.

\subsection{Expansion of the velocity potential}

When the cylinder is in oscillation with small amplitude, the boundary conditions in Eqs. (8) and (9) can be imposed on its mean position. As a result, the time factor exp(i $\omega t)$ can be taken out. At large amplitude, Eqs. (8) and (9) should be imposed on the instantaneous position of the body. Its sinusoidal motion with frequency $\omega$ will generate fluid motions of frequencies $n \omega \quad(n=1,2, \ldots)$. Thus, we can write the potentials $\phi_{j}$ in terms of multipole expansions in the polar coordinate system as

$$
\phi_{j}=\sum_{m=1}^{\infty} \sum_{s=-\infty}^{\infty} A_{m}^{s} a^{m}\left[\frac{\mathrm{e}^{\mathrm{i} m \theta+\mathrm{i} s \omega t}}{r^{m}}+f_{m}^{s}(r, \theta, t)\right]+\sum_{m=1}^{\infty} \sum_{s=-\infty}^{\infty} B_{m}^{s} a^{m}\left[\frac{\mathrm{e}^{-\mathrm{i} m \theta+\mathrm{i} s \omega t}}{r^{m}}+g_{m}^{s}(r, \theta, t)\right]
$$

where $f_{m}^{s}$ and $g_{m}^{s}$ are introduced to satisfy the boundary condition on the ice sheet. To do that, we 
$8 \quad$ where $v=\omega^{2} / g$.

use [18]

$$
\frac{\mathrm{e}^{ \pm \mathrm{i} m \theta+\mathrm{i} s \omega t}}{r^{m}}=\frac{1}{(m-1) !} \sum_{p=-\infty}^{\infty} \sum_{q=-\infty}^{\infty}(-1)^{q}( \pm \mathrm{i})^{q} \mathrm{e}^{\mathrm{i} p \alpha_{3}+\mathrm{i} q \alpha_{1}+\mathrm{i} s \omega t} \int_{0}^{\infty} k^{m-1} \mathrm{e}^{[-k(z+h) \pm \mathrm{i} k x]} I_{p}\left(k \eta_{3}\right) J_{q}\left(k \eta_{1}\right) \mathrm{d} k
$$

where $J_{q}\left(k \eta_{1}\right)$ and $I_{p}\left(k \eta_{3}\right)$ are the Bessel functions and the modified Bessel functions respectively. We may then write $f_{m}^{s}$ and $g_{m}^{s}$ in the following form

$$
f_{m}^{s}, g_{m}^{s}=\frac{1}{(m-1) !} \sum_{p=-\infty}^{\infty} \sum_{q=-\infty}^{\infty}(-1)^{q}( \pm \mathrm{i})^{q} e^{\mathrm{i} p \alpha_{3}+\mathrm{i} q \alpha_{1}+\mathrm{i} s \omega t} \int_{0}^{\infty} A(k) k^{m-1} \mathrm{e}^{[k(z-h) \pm \mathrm{i} k x]} I_{p}\left(k \eta_{3}\right) J_{q}\left(k \eta_{1}\right) \mathrm{d} k
$$

Substituting Eqs. (11) and (12) into Eq. (5), we obtain

$$
A(k)=\frac{k\left(D k^{4}-K v(p+q+s)^{2}+1\right)+v(p+q+s)^{2}}{k\left(D k^{4}-K v(p+q+s)^{2}+1\right)-v(p+q+s)^{2}}
$$

Then the potential $\phi_{j}$ in Eq. (10) can be written as

$$
\begin{aligned}
\phi_{j} & =\sum_{m=1}^{\infty} \sum_{s=-\infty}^{\infty} A_{m}^{s} a^{m}\left[\frac{e^{\mathrm{i} m \theta+\mathrm{i} s \omega t}}{r^{m}}+\frac{1}{(m-1) !} \sum_{p=-\infty}^{\infty} \sum_{q=-\infty}^{\infty}(-\mathrm{i})^{q} \mathrm{e}^{\mathrm{i} p \alpha_{3}+\mathrm{i} q \alpha_{1}+\mathrm{i} s \omega t}\right. \\
& \left.\times \int_{0}^{\infty} \frac{k\left(D k^{4}-K v(p+q+s)^{2}+1\right)+v(p+q+s)^{2}}{k\left(D k^{4}-K v(p+q+s)^{2}+1\right)-v(p+q+s)^{2}} k^{m-1} \mathrm{e}^{[k(z-h)+\mathrm{i} k x]} I_{p}\left(k \eta_{3}\right) J_{q}\left(k \eta_{1}\right) \mathrm{d} k\right] \\
& +\sum_{m=1}^{\infty} \sum_{s=-\infty}^{\infty} B_{m}^{s} a^{m}\left[\frac{e^{-\mathrm{i} m \theta+\mathrm{i} s \omega t}}{r^{m}}+\frac{1}{(m-1) !} \sum_{p=-\infty}^{\infty} \sum_{q=-\infty}^{\infty}(+\mathrm{i})^{q} \mathrm{e}^{\mathrm{i} p \alpha_{3}+\mathrm{i} q \alpha_{1}+\mathrm{i} s \omega t}\right. \\
& \left.\times \int_{0}^{\infty} \frac{k\left(D k^{4}-K v(p+q+s)^{2}+1\right)+v(p+q+s)^{2}}{k\left(D k^{4}-K v(p+q+s)^{2}+1\right)-v(p+q+s)^{2}} k^{m-1} \mathrm{e}^{[k(z-h)-\mathrm{i} k x]} I_{p}\left(k \eta_{3}\right) J_{q}\left(k \eta_{1}\right) \mathrm{d} k\right]
\end{aligned}
$$

where the integration route passes over the pole $k=\lambda$ with $p+q+s>0$ and passes under the pole $k=\lambda$ with $p+q+s<0$ to satisfy the outgoing waves condition at $x \rightarrow \pm \infty$. Here $\lambda$ is the only real positive root of the dispersion equation

$$
k\left[D k^{4}-K v(p+q+s)^{2}+1\right]-v(p+q+s)^{2}=0
$$

at each given $p+q+s$. Eq. (14) then satisfies the governing Laplace equation and the boundary conditions on the ice sheet and at infinity. The remaining boundary condition is that on the body surface. To satisfy this remaining condition, we use [18]

$$
\mathrm{e}^{k(z-h) \pm \mathrm{i} k x}=\mathrm{e}^{-2 k h} \sum_{n=0}^{\infty} \frac{k^{n} r^{n} \mathrm{e}^{ \pm \mathrm{i} n \theta}}{n !} \sum_{p_{1}=-\infty}^{\infty} \sum_{q_{1}=-\infty}^{\infty}( \pm \mathrm{i})^{q_{1}} I_{p_{1}}\left(k \eta_{3}\right) J_{q_{1}}\left(k \eta_{1}\right) \mathrm{e}^{\mathrm{i} q_{1} \alpha_{1}} \mathrm{e}^{\mathrm{i} p_{1} \alpha_{3}}
$$

to expand Eq. (14) in the polar coordinate system as

$$
\begin{aligned}
\phi_{j} & =\sum_{m=1}^{\infty} \sum_{s=-\infty}^{\infty} A_{m}^{s} a^{m}\left[\frac{e^{\mathrm{i} m \theta+\mathrm{i} s \omega t}}{r^{m}}+\frac{1}{(m-1) !} \sum_{n=0}^{\infty} \frac{r^{n} e^{+\mathrm{i} n \theta}}{n !} \sum_{p=-\infty}^{\infty} \sum_{q=-\infty}^{\infty} \sum_{p_{1}=-\infty}^{\infty} \sum_{q_{1}=-\infty}^{\infty}\right. \\
& \left.\times(-\mathrm{i})^{q}(+\mathrm{i})^{q_{1}} \exp \left(\mathrm{i} p \alpha_{3}+\mathrm{i} q \alpha_{1}+\mathrm{i} s \omega t+\mathrm{i} q_{1} \alpha_{1}+\mathrm{i} p_{1} \alpha_{3}\right) F\left(m, n, p, q, p_{1}, q_{1}, p+q+s\right)\right] \\
& +\sum_{m=1}^{\infty} \sum_{s=-\infty}^{\infty} B_{m}^{s} a^{m}\left[\frac{e^{-\mathrm{i} m \theta+\mathrm{i} s \omega t}}{r^{m}}+\frac{1}{(m-1) !} \sum_{n=0}^{\infty} \frac{r^{n} e^{-\mathrm{i} n \theta}}{n !} \sum_{p=-\infty}^{\infty} \sum_{q=-\infty}^{\infty} \sum_{p_{1}=-\infty}^{\infty} \sum_{q_{1}=-\infty}^{\infty}\right. \\
& \left.\times(+\mathrm{i})^{q}(-\mathrm{i})^{q_{1}} \exp \left(\mathrm{i} p \alpha_{3}+\mathrm{i} q \alpha_{1}+\mathrm{i} s \omega t+\mathrm{i} q_{1} \alpha_{1}+\mathrm{i} p_{1} \alpha_{3}\right) F\left(m, n, p, q, p_{1}, q_{1}, p+q+s\right)\right]
\end{aligned}
$$

where 


$$
\begin{aligned}
F\left(m, n, p, q, p_{1}, q_{1}, s\right)=\int_{0}^{\infty} \frac{k\left(D k^{4}-K v s^{2}+1\right)+v s^{2}}{k\left(D k^{4}-K v s^{2}+1\right)-v s^{2}} \\
\quad \times k^{m+n-1} \mathrm{e}^{-2 k h} I_{p_{1}}\left(k \eta_{3}\right) J_{q_{1}}\left(k \eta_{1}\right) I_{p}\left(k \eta_{3}\right) J_{q}\left(k \eta_{1}\right) \mathrm{d} k
\end{aligned}
$$

2 Invoking the boundary conditions in Eqs. (8) and (9), then using the orthogonality of

3 trigonometric function, we have for $\phi_{1}$

4

$$
\begin{aligned}
-m \frac{A_{m}^{s}}{a}+ & \sum_{n=1}^{\infty} \frac{a^{n+m-1}}{(n-1) !(m-1) !} \sum_{p=-\infty}^{\infty} \sum_{q=-\infty}^{\infty} \sum_{p_{1}=-\infty}^{\infty} \sum_{u=-\infty}^{\infty} \\
& \times(-\mathrm{i})^{q}(+\mathrm{i})^{q_{1}} \mathrm{e}^{\mathrm{i}\left(q+q_{1}\right) \gamma_{1} \mathrm{i}\left(p+p_{1}\right) \gamma_{3}} F\left(n, m, p, q, p_{1}, q_{1}, p+q+u\right) A_{n}^{u}=-\frac{1}{2} \delta(s-1) \delta(m-1) \\
-m \frac{B_{m}^{s}}{a}+ & \sum_{n=1}^{\infty} \frac{a^{n+m-1}}{(n-1) !(m-1) !} \sum_{p=-\infty}^{\infty} \sum_{q=-\infty}^{\infty} \sum_{p_{1}=-\infty}^{\infty} \sum_{u=-\infty}^{\infty} \\
& \times(+\mathrm{i})^{q}(-\mathrm{i})^{q_{1}} \mathrm{e}^{\mathrm{i}\left(q+q_{1}\right) \gamma_{1}+\mathrm{i}\left(p+p_{1}\right) \gamma_{3}} F\left(n, m, p, q, p_{1}, q_{1}, p+q+u\right) B_{n}^{u}=\frac{1}{2} \delta(s-1) \delta(m-1)
\end{aligned}
$$

5

where $u=s-q-q_{1}-p-p_{1}$, and $\delta(s-1)=1$ if $s=1$ and $\delta(s-1)=0$ otherwise. Similarly, we 6

7

$$
\begin{aligned}
-m \frac{B_{m}^{s}}{a} & +\sum_{n=1}^{\infty} \frac{a^{n+m-1}}{(n-1) !(m-1) !} \sum_{p=-\infty}^{\infty} \sum_{q=-\infty}^{\infty} \sum_{p_{1}=-\infty}^{\infty} \sum_{u=-\infty}^{\infty} \\
& \times(+\mathrm{i})^{q}(-\mathrm{i})^{q_{1}} \mathrm{e}^{\mathrm{i}\left(q+q_{1}\right) \gamma_{1}+\mathrm{i}\left(p+p_{1}\right) \gamma_{3}} F\left(n, m, p, q, p_{1}, q_{1}, p+q+u\right) B_{n}^{u}=-\frac{\mathrm{i}}{2} \delta(s-1) \delta(m-1)
\end{aligned}
$$

The comparison of Eq. (19) with Eq. (20) yields

$$
A_{m}^{s}(3)=\mathrm{i} A_{m}^{s}(1) ; B_{m}^{s}(3)=-\mathrm{i} B_{m}^{s}(1)
$$

where $j$ in $A_{m}^{s}(j)$ and $B_{m}^{s}(j)$ indicates that the coefficients correspond to $\phi_{j}$.

Substituting Eqs. (19) and (20) into Eq. (17), and let $r=a$, we have

$$
\begin{aligned}
& \phi_{1}=2 \sum_{m=1}^{\infty} \sum_{s=-\infty}^{\infty}\left[A_{m}^{s}(1) \mathrm{e}^{\mathrm{i} m \theta}+B_{m}^{s}(1) \mathrm{e}^{-\mathrm{i} m \theta}\right] \mathrm{e}^{\mathrm{i} s \omega t}-\mathrm{i} a \mathrm{e}^{\mathrm{i} \omega t} \sin \theta+\phi_{c 1}(t) \\
& \phi_{3}=2 \sum_{m=1}^{\infty} \sum_{s=-\infty}^{\infty}\left[A_{m}^{s}(3) \mathrm{e}^{\mathrm{i} m \theta}+B_{m}^{s}(3) \mathrm{e}^{-\mathrm{i} m \theta}\right] \mathrm{e}^{\mathrm{i} s \omega t}-\mathrm{i} a \mathrm{e}^{\mathrm{i} \omega t} \cos \theta+\phi_{c 3}(t)
\end{aligned}
$$

where $\phi_{c 1}(t)$ and $\phi_{c 3}(t)$ are functions of time only and they arise from the term $n=0$ in Eq. (17) and can be given as

$$
\begin{aligned}
\phi_{c 1}(t)=\sum_{m=1}^{\infty} \sum_{s=-\infty}^{\infty} \sum_{p=-\infty}^{\infty} \sum_{q=-\infty}^{\infty} \sum_{p_{1}=-\infty}^{\infty} \sum_{q_{1}=-\infty}^{\infty}\left[\frac{a^{m}}{(m-1) !}\left(A_{m}^{s}(1)(-\mathrm{i})^{q}(+\mathrm{i})^{q_{1}}+B_{m}^{s}(1)(+\mathrm{i})^{q}(-\mathrm{i})^{q_{1}}\right)\right. \\
\left.\quad \times \exp \left(\mathrm{i} p \alpha_{3}+\mathrm{i} q \alpha_{1}+\mathrm{i} s \omega t+\mathrm{i} q_{1} \alpha_{1}+\mathrm{i} p_{1} \alpha_{3}\right) F\left(m, 0, p, q, p_{1}, q_{1}, p+q+s\right)\right] \\
\phi_{c 3}(t)=\sum_{m=1}^{\infty} \sum_{s=-\infty}^{\infty} \sum_{p=-\infty}^{\infty} \sum_{q=-\infty}^{\infty} \sum_{p_{1}=-\infty}^{\infty} \sum_{q_{1}=-\infty}^{\infty}\left[\frac{a^{m}}{(m-1) !}\left(A_{m}^{s}(3)(-\mathrm{i})^{q}(+\mathrm{i})^{q_{1}}+B_{m}^{s}(3)(+\mathrm{i})^{q}(-\mathrm{i})^{q_{1}}\right)\right. \\
\left.\quad \times \exp \left(\mathrm{i} p \alpha_{3}+\mathrm{i} q \alpha_{1}+\mathrm{i} s \omega t+\mathrm{i} q_{1} \alpha_{1}+\mathrm{i} p_{1} \alpha_{3}\right) F\left(m, 0, p, q, p_{1}, q_{1}, p+q+s\right)\right]
\end{aligned}
$$

Then invoking Eq. (7), we obtain 


$$
\Phi=2 \operatorname{Re}\left[\sum_{m=1}^{\infty} \sum_{s=-\infty}^{\infty}\left(C_{m}^{s} \mathrm{e}^{\mathrm{i} m \theta}+D_{m}^{s} \mathrm{e}^{-\mathrm{i} m \theta}\right) \mathrm{e}^{\mathrm{i} s \omega t}\right]-\omega a \eta_{1} \sin \alpha_{1} \sin \theta-\omega a \eta_{3} \sin \alpha_{3} \cos \theta+\Phi_{c}(t)
$$

where

$$
\begin{aligned}
& C_{m}^{s}=-A_{m}^{s}(1) \omega \eta_{1} \mathrm{e}^{\mathrm{i} \gamma_{1}}-A_{m}^{s}(3) \omega \eta_{3} \mathrm{e}^{\mathrm{i} \gamma_{3}}=-\omega\left(\eta_{1} \mathrm{e}^{\mathrm{i} \gamma_{1}}+\mathrm{i} \eta_{3} \mathrm{e}^{\mathrm{i} / \gamma_{3}}\right) A_{m}^{s}(1) \\
& D_{m}^{s}=-B_{m}^{s}(1) \omega \eta_{1} \mathrm{e}^{\mathrm{i} \gamma_{1}}-B_{m}^{s}(3) \omega \eta_{3} \mathrm{e}^{\mathrm{i} / 3}=-\omega\left(\eta_{1} \mathrm{e}^{\mathrm{i} \gamma_{1}}-\mathrm{i} \eta_{3} \mathrm{e}^{\mathrm{i} / 3}\right) B_{m}^{s}(1)
\end{aligned}
$$

and

$$
\Phi_{c}(t)=-\omega \eta_{1} \operatorname{Re}\left[\phi_{c 1}(t) \mathrm{e}^{\mathrm{i} / \gamma_{1}}\right]-\omega \eta_{3} \operatorname{Re}\left[\phi_{c 3}(t) \mathrm{e}^{\mathrm{i} / \gamma_{3}}\right]
$$

\subsection{The hydrodynamic force}

When the problem for the velocity potential has been solved, the hydrodynamic force $F_{j}$ can be found by direct integration of the pressure obtained from the Bernoulli equation over the surface of the cylinder, which can be equivalently written as [18]

$$
F_{j}=-\frac{d}{d t} \int_{S_{0}} \rho \Phi n_{j} \mathrm{~d} s
$$

$$
-\frac{\rho}{2} \int_{S_{0}} \nabla\left(\Phi+\omega \eta_{1} x \sin \alpha_{1}+\omega \eta_{3} z \sin \alpha_{3}\right) \cdot \nabla\left(\Phi+\omega \eta_{1} x \sin \alpha_{1}+\omega \eta_{3} z \sin \alpha_{3}\right) n_{j} \mathrm{~d} s
$$

The substitution of Eq. (26) into Eq. (29) yields

$$
F_{j}=\operatorname{Re}\left[\sum_{s=-\infty}^{\infty} F_{j}(s) \mathrm{e}^{\mathrm{i} s \omega t}\right]
$$

where the horizontal force components $F_{1}(s)$ and vertical force components $F_{3}(s)$ are given as follow

$$
\begin{aligned}
F_{1}(s) & =-\frac{1}{2} \rho \pi \omega^{2} a^{2} \eta_{1} \mathrm{e}^{\mathrm{i} s \gamma_{1}}[\delta(s-1)+\delta(s+1)]+\rho \pi\left\{2 a s \omega\left(-C_{1}^{s}+D_{1}^{s}\right)\right. \\
& +\frac{\mathrm{i}}{a} \sum_{m=1}^{\infty} \sum_{s_{1}=-\infty}^{\infty}\left[m ( m + 1 ) \left(C_{m+1}^{s-s_{1}} D_{m}^{s_{1}}-D_{m+1}^{s-s_{1}} C_{m}^{s_{1}}-C_{m}^{s-s_{1}} D_{m+1}^{s_{1}}+D_{m}^{s-s_{1}} C_{m+1}^{s_{1}}\right.\right. \\
& \left.\left.\left.+C_{m+1}^{s+s_{1}} \bar{C}_{m}^{s_{1}}-D_{m+1}^{s+s_{1}} \bar{D}_{m}^{s_{1}}-C_{m}^{s+s_{1}} \bar{C}_{m+1}^{s_{1}}+D_{m}^{s+s_{1}} \bar{D}_{m+1}^{s_{1}}\right)\right]\right\}
\end{aligned}
$$

and

$$
\begin{aligned}
F_{3}(s) & =-\frac{1}{2} \rho \pi \omega^{2} a^{2} \eta_{3} \mathrm{e}^{\mathrm{i} \gamma_{3}}[\delta(s-1)+\delta(s+1)]+\rho \pi\left\{2 a \mathrm{i} s \omega\left(C_{1}^{s}+D_{1}^{s}\right)\right. \\
& +\frac{1}{a} \sum_{m=1}^{\infty} \sum_{s_{1}=-\infty}^{\infty}\left[m ( m + 1 ) \left(C_{m+1}^{s-s_{1}} D_{m}^{s_{1}}+D_{m+1}^{s-s_{1}} C_{m}^{s_{1}}+C_{m}^{s-s_{1}} D_{m+1}^{s_{1}}+D_{m}^{s-s_{1}} C_{m+1}^{s_{1}}\right.\right. \\
& \left.\left.\left.+C_{m+1}^{s+s_{1}} \bar{C}_{m}^{s_{1}}+D_{m+1}^{s+s_{1}} \bar{D}_{m}^{s_{1}}+C_{m}^{s+s_{1}} \bar{C}_{m+1}^{s_{1}}+D_{m}^{s+s_{1}} \bar{D}_{m+1}^{s_{1}}\right)\right]\right\}
\end{aligned}
$$

We may also write Eq. (30) as

$$
F_{j}=\operatorname{Re}\left\{F_{j}(0)+\sum_{s=1}^{\infty}\left[F_{j}(s) \mathrm{e}^{\mathrm{i} s \omega t}+F_{j}(-s) \mathrm{e}^{-\mathrm{i} s \omega t}\right]\right\}=\operatorname{Re}\left\{F_{j}(0)+\sum_{s=1}^{\infty}\left[F_{j}(s)+\bar{F}_{j}(-s)\right] \mathrm{e}^{\mathrm{i} s \omega t}\right\}
$$

where the over-bar denotes conjugation. The non-dimensional force amplitudes $c_{j}(s)$ are then defined as

$$
c_{j}(0)=\operatorname{Re}\left[F_{j}(0)\right] / \rho \pi \omega^{2} a^{2} \eta_{i} ; c_{j}(s)=\left|F_{j}(s)+\bar{F}_{j}(-s)\right| / \rho \pi \omega^{2} a^{2} \eta_{i} \text { if } s>0
$$

We notice that the horizontal steady force $F_{1}(0)$ can be also computed by the far field method (see appendix), which can be used to verify the formulation and solution procedure. 
2 The deformation of the ice sheet $w(x, t)$ can be obtained by the integration of the kinematic 3 boundary condition with respect to time, or

$$
w(x, t)=\left.\int \Phi_{z}\right|_{\mathrm{z}=0} \mathrm{~d} t
$$

Through Eqs. (7), (11), (13) and (14), we have

$$
w(x, t)=\operatorname{Re}[W(x, t)]+f(x)
$$

where $f(x)$ is a time independent function and

$$
\begin{aligned}
W(x, t)= & \sum_{u=-\infty}^{\infty} \frac{\mathrm{e}^{\mathrm{i} u \omega t}}{\mathrm{i} u \omega} \sum_{m=1}^{\infty} \frac{a^{m}}{(m-1) !} \sum_{s=-\infty}^{\infty} C_{m}^{s} \\
& \times \sum_{p=-\infty}^{\infty}(-\mathrm{i})^{q} \mathrm{e}^{\mathrm{i} p \gamma_{3}+\mathrm{i} \gamma \alpha_{1}} \int_{0}^{\infty}[A(k)-1] \mathrm{e}^{-k h+\mathrm{i} k x} k^{m} I_{p}\left(k \eta_{3}\right) J_{q}\left(k \eta_{1}\right) \mathrm{d} k \\
+ & \sum_{u=-\infty}^{\infty} \frac{\mathrm{e}^{\mathrm{i} u \omega t}}{\mathrm{i} u \omega} \sum_{m=1}^{\infty} \frac{a^{m}}{(m-1) !} \sum_{s=-\infty}^{\infty} D_{m}^{s} \\
& \times \sum_{p=-\infty}^{\infty}(+\mathrm{i})^{q} \mathrm{e}^{\mathrm{i} p \gamma_{3}+\mathrm{i} \gamma \alpha_{1}} \int_{0}^{\infty}[A(k)-1] \mathrm{e}^{-k h-\mathrm{i} k x} k^{m} I_{p}\left(k \eta_{3}\right) J_{q}\left(k \eta_{1}\right) \mathrm{d} k
\end{aligned}
$$
with $q=u-p-s$. The summation in Eq. (37) excludes the term of $u=0$, as this term does not appear in the series of $\Phi_{z}$. From the dynamic condition on the ice sheet, it can also be confirmed that $f(x)=0$.

Let $x \rightarrow \pm \infty$ in Eq. (37), we obtain the asymptotic expressions for the deformation of the ice sheet

$$
\begin{aligned}
\lim _{x \rightarrow \pm \infty} W=\sum_{u=-\infty}^{\infty} \frac{\mathrm{e}^{\mathrm{i} u \omega t}}{u \omega} \sum_{m=1}^{\infty} \frac{a^{m}}{(m-1) !} \sum_{s=-\infty}^{\infty} C_{m}^{s} \sum_{p=-\infty}^{\infty}(-\mathrm{i})^{q} \mathrm{e}^{\mathrm{i} p \gamma_{3}+\mathrm{i} q \gamma_{1}} \\
\times[ \pm 1-\operatorname{sgn}(u)] \pi \frac{\mathrm{e}^{\mathrm{i} \lambda x} \mathrm{e}^{-\lambda h} \lambda^{m} I_{p}\left(\lambda \eta_{3}\right) J_{q}\left(\lambda \eta_{1}\right) \alpha(\lambda, u)}{\beta^{\prime}(\lambda, u)} \\
+\sum_{u=-\infty}^{\infty} \frac{\mathrm{e}^{\mathrm{i} u \omega t}}{u \omega} \sum_{m=1}^{\infty} \frac{a^{m}}{(m-1) !} \sum_{s=-\infty}^{\infty} D_{m}^{s} \sum_{p=-\infty}^{\infty}(+\mathrm{i})^{q} \mathrm{e}^{\mathrm{i} p \gamma_{3}+\mathrm{i} q \gamma_{1}} \\
\times[\mp 1-\operatorname{sgn}(u)] \pi \frac{\mathrm{e}^{-\mathrm{i} \lambda x} \mathrm{e}^{-\lambda h} \lambda^{m} I_{p}\left(\lambda \eta_{3}\right) J_{q}\left(\lambda \eta_{1}\right) \alpha(\lambda, u)}{\beta^{\prime}(\lambda, u)}
\end{aligned}
$$

where $\alpha(\lambda, u)$ and $\beta(\lambda, u)$ are defined in appendix Eq. (68).

\subsection{Some typical cases}

Here, we will consider four typical cases, i.e. linear case, purely horizontal motion case, purely vertical motion case, and circular motion case, which have been considered previously by $\mathrm{Wu}$ [18] for the free surface condition.

\subsubsection{Linear case}

When the motion amplitudes are sufficiently small, we can linearize the body surface boundary condition at its mean position. Thus, we can set $\eta_{1}=\eta_{3}=0$ in the expansion of $\phi_{j}$, which means that we need to retain only the terms of $p=p_{1}=q=q_{1}=0$ in Eqs. (19) and (20). Then we have 
for $\phi_{1}$

and for $\phi_{3}$

$$
\begin{aligned}
& -m \frac{A_{m}^{1}}{a}+\frac{1}{(m-1) !} \sum_{n=1}^{\infty} \frac{a^{n+m-1}}{(n-1) !} F(n, m, 0,0,0,0,1) A_{n}^{1}=-\frac{1}{2} \delta(m-1) \\
& B_{m}^{s}=-A_{m}^{1}
\end{aligned}
$$

$$
\begin{aligned}
& -m \frac{A_{m}^{1}}{a}+\frac{1}{(m-1) !} \sum_{n=1}^{\infty} \frac{a^{n+m-1}}{(n-1) !} F(n, m, 0,0,0,0,1) A_{n}^{1}=-\frac{\mathrm{i}}{2} \delta(m-1) \\
& B_{m}^{s}=A_{m}^{1}
\end{aligned}
$$

The above two equations suggest that $\phi_{1}$ in Eq. (14) is antisymmetric about $\theta=0$ or $x=0$, and $\phi_{3}$ in Eq. (14) is symmetric about $\theta=0$ and $F_{1}=0$ in Eq. (31).

Similarly, we need to retain only the terms of $p=q=0$ and $s=1$ for the deformation of the ice sheet. Thus

$$
\begin{aligned}
W & =\frac{\mathrm{e}^{\mathrm{i} \omega t}}{\mathrm{i} \omega} \sum_{m=1}^{\infty} \frac{C_{m}^{1} a^{m}}{(m-1) !} \int_{0}^{\infty}[A(k)-1] \mathrm{e}^{-k h+\mathrm{i} k x} k^{m} \mathrm{~d} k \\
& +\frac{\mathrm{e}^{\mathrm{i} \omega t}}{\mathrm{i} \omega} \sum_{m=1}^{\infty} \frac{D_{m}^{1} a^{m}}{(m-1) !} \int_{0}^{\infty}[A(k)-1] \mathrm{e}^{-k h-\mathrm{i} k x} k^{m} \mathrm{~d} k
\end{aligned}
$$

and

$$
\begin{aligned}
& \lim _{x \rightarrow+\infty} W=-2 \pi \frac{\mathrm{e}^{\mathrm{i} \omega t}}{\omega} \frac{\mathrm{e}^{-\mathrm{i} \lambda x} \mathrm{e}^{-\lambda h} \alpha(\lambda, 1)}{\beta^{\prime}(\lambda, 1)} \sum_{m=1}^{\infty} \frac{D_{m}^{1} a^{m} \lambda^{m}}{(m-1) !} \\
& \lim _{x \rightarrow-\infty} W=-2 \pi \frac{\mathrm{e}^{\mathrm{i} \omega t}}{\omega} \frac{\mathrm{e}^{\mathrm{i} \lambda x} \mathrm{e}^{-\lambda h} \alpha(\lambda, 1)}{\beta^{\prime}(\lambda, 1)} \sum_{m=1}^{\infty} \frac{C_{m}^{1} a^{m} \lambda^{m}}{(m-1) !}
\end{aligned}
$$

\subsubsection{Purely horizontal motion}

When $\eta_{3}=0$, we need to retain only the terms of $p=p_{1}=0$ in Eq. (19). We have

$$
-m \frac{A_{m}^{s}}{a}+\frac{1}{(m-1) !} \sum_{n=1}^{\infty} \frac{a^{n+m-1}}{(n-1) !} \sum_{q=-\infty}^{\infty} \sum_{u=-\infty}^{\infty}(-1)^{q}(+\mathrm{i})^{s-u} \mathrm{e}^{\mathrm{i}(s-u) \gamma_{1}}
$$

$$
\times F(n, m, 0, q, 0, s-q-u, q+u) A_{n}^{u}=-\frac{1}{2} \delta(s-1) \delta(m-1)
$$

$$
B_{m}^{s}=(-1)^{s} A_{m}^{s}
$$

These two equations means that $F_{1}(s)=0$ when $s=2 n \omega$, and $F_{3}(s)=0$ when $s=(2 n+1) \omega$, when Eqs. (27), (31) and (32) are used.

Similarly, we retain the terms $p=0$ for the deformation of the ice sheet, thus we obtain

$$
\begin{aligned}
W & =\sum_{u=-\infty}^{\infty} \frac{\mathrm{e}^{\mathrm{i} u \omega t}}{\mathrm{i} u \omega} \sum_{m=1}^{\infty} \frac{a^{m}}{(m-1) !} \sum_{s=-\infty}^{\infty} C_{m}^{s}(-\mathrm{i})^{q} \mathrm{e}^{\mathrm{i} q \gamma_{1}} \int_{0}^{\infty}[A(k)-1] \mathrm{e}^{-k h+\mathrm{i} k x} k^{m} J_{q}\left(k \eta_{1}\right) \mathrm{d} k \\
& +\sum_{u=-\infty}^{\infty} \frac{\mathrm{e}^{\mathrm{i} u \omega t}}{\mathrm{i} u \omega} \sum_{m=1}^{\infty} \frac{a^{m}}{(m-1) !} \sum_{s=-\infty}^{\infty} D_{m}^{s}(+\mathrm{i})^{q} \mathrm{e}^{\mathrm{i} q \gamma_{1}} \int_{0}^{\infty}[A(k)-1] \mathrm{e}^{-k h-\mathrm{i} k x} k^{m} J_{q}\left(k \eta_{1}\right) \mathrm{d} k
\end{aligned}
$$

and 


$$
\begin{aligned}
\lim _{x \rightarrow \pm \infty} W=\sum_{u=-\infty}^{\infty} \frac{\mathrm{e}^{\mathrm{i} u \omega t}}{u \omega} \sum_{m=1}^{\infty} \frac{a^{m}}{(m-1) !} \sum_{s=-\infty}^{\infty} C_{m}^{s}(-\mathrm{i})^{q} \mathrm{e}^{\mathrm{i} q \gamma_{1}} \\
\times[ \pm 1-\operatorname{sgn}(u)] \pi \frac{\mathrm{e}^{\mathrm{i} \lambda x} \mathrm{e}^{-\lambda h} \lambda^{m} J_{q}\left(\lambda \eta_{1}\right) \alpha(\lambda, u)}{\beta^{\prime}(\lambda, u)}
\end{aligned}
$$

$$
\begin{aligned}
+\sum_{u=-\infty}^{\infty} \frac{\mathrm{e}^{\mathrm{i} u \omega t}}{u \omega} \sum_{m=1}^{\infty} \frac{a^{m}}{(m-1) !} \sum_{s=-\infty}^{\infty} D_{m}^{s}(+\mathrm{i})^{q} \mathrm{e}^{\mathrm{i} q \gamma_{1}} \\
\times[\mp 1-\operatorname{sgn}(u)] \pi \frac{\mathrm{e}^{-\mathrm{i} \lambda x} \mathrm{e}^{-\lambda h} \lambda^{m} J_{q}\left(\lambda \eta_{1}\right) \alpha(\lambda, u)}{\beta^{\prime}(\lambda, u)}
\end{aligned}
$$

\subsubsection{Purely vertical motion}

When $\eta_{1}=0$, we need to retain only the terms of $q=q_{1}=0$ in Eq. (20). We have

$$
\begin{aligned}
-m \frac{A_{m}^{s}}{a}+\frac{1}{(m-1) !} \sum_{n=1}^{\infty} \frac{a^{n+m-1}}{(n-1) !} \sum_{p=-\infty}^{\infty} \sum_{u=-\infty}^{\infty} \mathrm{e}^{\mathrm{i}(s-u) \gamma_{3}} F(n, m, p, 0, s- & p-u, 0, p+u) A_{n}^{u} \\
& =-\frac{\mathrm{i}}{2} \delta(s-1) \delta(m-1)
\end{aligned}
$$

$$
B_{m}^{s}=A_{m}^{s}
$$

5 These two equations imply that $\phi_{3}$ in Eq. (14) is symmetric about $\theta=0$ and $F_{1}=0$ in Eq. (31).

6 Similarly, we retain only the terms $q=0$ for the deformation of the ice sheet. Thus we obtain

$$
\begin{aligned}
W & =\sum_{u=-\infty}^{\infty} \frac{\mathrm{e}^{\mathrm{i} u \omega t}}{\mathrm{i} u \omega} \sum_{m=1}^{\infty} \frac{a^{m}}{(m-1) !} \sum_{s=-\infty}^{\infty} C_{m}^{s} \sum_{p=-\infty}^{\infty} \mathrm{e}^{\mathrm{i} p \gamma_{3}} \int_{0}^{\infty}[A(k)-1] \mathrm{e}^{-k h+\mathrm{i} k x} k^{m} I_{p}\left(k \eta_{3}\right) \mathrm{d} k \\
& +\sum_{u=-\infty}^{\infty} \frac{\mathrm{e}^{\mathrm{i} u \omega t}}{\mathrm{i} u \omega} \sum_{m=1}^{\infty} \frac{a^{m}}{(m-1) !} \sum_{s=-\infty}^{\infty} D_{m}^{s} \sum_{p=-\infty}^{\infty} \mathrm{e}^{\mathrm{i} p \gamma_{3}} \int_{0}^{\infty}[A(k)-1] \mathrm{e}^{-k h-\mathrm{i} k x} k^{m} I_{p}\left(k \eta_{3}\right) \mathrm{d} k
\end{aligned}
$$

and

$$
\begin{aligned}
\lim _{x \rightarrow \pm \infty} W=\sum_{u=-\infty}^{\infty} \frac{\mathrm{e}^{\mathrm{i} u \omega t}}{u \omega} \sum_{m=1}^{\infty} \frac{a^{m}}{(m-1) !} \sum_{s=-\infty}^{\infty} C_{m}^{s} \sum_{p=-\infty}^{\infty} \mathrm{e}^{\mathrm{i} p \gamma_{3}} \\
\times[ \pm 1-\operatorname{sgn}(u)] \pi \frac{\mathrm{e}^{\mathrm{i} \lambda x} \mathrm{e}^{-\lambda h} \lambda^{m} I_{p}\left(\lambda \eta_{3}\right) \alpha(\lambda, u)}{\beta^{\prime}(\lambda, u)} \\
+\sum_{u=-\infty}^{\infty} \frac{\mathrm{e}^{\mathrm{i} u \omega t}}{u \omega} \sum_{m=1}^{\infty} \frac{a^{m}}{(m-1) !} \sum_{s=-\infty}^{\infty} D_{m}^{s} \sum_{p=-\infty}^{\infty} \mathrm{e}^{\mathrm{i} p \gamma_{3}} \\
\times[\mp 1-\operatorname{sgn}(u)] \pi \frac{\mathrm{e}^{-\mathrm{i} \lambda x} \mathrm{e}^{-\lambda h} \lambda^{m} I_{p}\left(\lambda \eta_{3}\right) \alpha(\lambda, u)}{\beta^{\prime}(\lambda, u)}
\end{aligned}
$$

\subsubsection{Circular motion}

When $\eta_{1}=\eta_{3}=\eta$ and $\gamma_{3}=\gamma_{1} \pm \pi / 2=\gamma$, the cylinder moves in a circular path with the centre at $(0,-h)$, where the positive sign means the motion is clockwise, while the negative sign means counterclockwise. Without losing generality, $\gamma$ can be set to be zero. Here we may also adopt the special form of multipole expansions for circular motion case [18]. We notice that Eq. (6) becomes

$$
\frac{\partial \Phi}{\partial r}=-\omega \eta \sin [\omega t-( \pm) \theta]
$$


We may define

$$
\Phi=-\omega \eta \operatorname{Re}(\phi)
$$

Then the body surface boundary condition becomes

$$
\partial \phi / \partial r=-\mathrm{ie}^{\mathrm{i}[\omega t-( \pm) \theta]}
$$

Taking clockwise motion as an example, the multipole expansion can be given as

$$
\phi=\sum_{m=1}^{\infty} \sum_{s=-\infty}^{\infty} B_{m}^{s} a^{m}\left[\frac{\mathrm{e}^{-\mathrm{i} m \theta+\mathrm{i} s \omega t}}{r^{m}}+\frac{1}{(m-1) !} \sum_{p=0}^{\infty} \frac{\eta^{p}}{p !} \mathrm{e}^{\mathrm{i} \rho \omega t+\mathrm{i} s \omega t} \int_{0}^{\infty} A(k) k^{m+p-1} \exp (k z-k h-\mathrm{i} k x) \mathrm{d} k\right]
$$

Invoking the upper surface condition, we have

$$
A(k)=\frac{k\left[D k^{4}-K v(p+s)^{2}+1\right]+v(p+s)^{2}}{k\left[D k^{4}-K v(p+s)^{2}+1\right]-v(p+s)^{2}}
$$

Eq. (53) is noticeably simpler than (14), which means the subsequent solution procedure is much simpler. In fact, the equation for the coefficients can be obtained as

$$
A_{m}^{s}=0
$$

$$
-\frac{m}{a} B_{m}^{s}+\frac{1}{(m-1) !} \sum_{n=1}^{\infty} \sum_{u=-\infty}^{\infty} \frac{a^{n+m-1}}{(n-1) !} \sum_{p=0}^{\infty} \frac{\eta^{p+p_{1}}}{p ! p_{1} !} F\left(m, n, p, p_{1}, p+u\right) B_{n}^{u}=-\mathrm{i} \delta(s-1) \delta(m-1)
$$

where $p_{1}=u-s-p$.

For the deformation of the ice sheet, we have

$$
W=-\sum_{m=1}^{\infty} \frac{a^{m}}{(m-1) !} \sum_{s=-\infty}^{\infty} B_{m}^{s} \sum_{p=0}^{\infty} \frac{\eta^{p+1}}{p !} \frac{\mathrm{e}^{\mathrm{i} p \omega t+\mathrm{i} s \omega t}}{\mathrm{i}(p+s)} \int_{0}^{\infty}(A(k)-1) k^{m+p} \mathrm{e}^{-k h-\mathrm{i} k x} \mathrm{~d} k
$$

This gives

$$
\lim _{x \rightarrow \pm \infty} W=-\sum_{m=1}^{\infty} \frac{a^{m}}{(m-1) !} \sum_{s=-\infty}^{\infty} B_{m}^{s} \sum_{p=0}^{\infty} \frac{\eta^{p+1}}{p !} \frac{\mathrm{e}^{\mathrm{i} p \omega t+\mathrm{i} s \omega t}}{(p+s)}
$$

$$
\times[\mp 1-\operatorname{sgn}(p+s)] \pi \frac{\mathrm{e}^{-\mathrm{i} \lambda x} \mathrm{e}^{-\lambda h} \lambda^{m+p} \alpha(\lambda, p+s)}{\beta^{\prime}(\lambda, p+s)}
$$

\section{Numerical results and discussions}

In the following numerical results and discussions, we will use dimensionless variables. The basic parameters are chosen as radius of the circular cylinder $a$, density of the water $\rho$ and gravitational acceleration $g$. The other parameters are nondimensionalized as

$$
h / a, D / a^{4}, K / a
$$

It should be noted that the infinite series is truncated at a finite number, i.e. the numerical results are obtained by taking $m=M$ and $s=S$, as their upper limits respectively.

\subsection{Analysis of the ice sheet effects on dispersion relationship}

Wave property is very much affected by its dispersion relationship. For the free surface flow, this relationship is in a simple form, especially in deep water. For the ice sheet, such a relationship in Eq. (15) is more complicated. Here we take $p+q+s=1$ as an example to conduct the analysis, which corresponds to the first time harmonic component, or

$$
k_{1}=v /\left(D k_{1}^{4}-K v+1\right)
$$


1 We can consider some typical values of ice sheet $E=5 \times 10^{9} \mathrm{~Pa}, \rho_{1}=922.5 \mathrm{~kg} / \mathrm{m}^{3}, v=0.3$ for a 2 cylinder $a=10 \mathrm{~m}$ with ice thickness varying from $h_{1}=0.5 \mathrm{~m}$ to $2 \mathrm{~m}$. The other parameters are $3 \rho=1025 \mathrm{~kg} / \mathrm{m}^{3}, g=9.81 \mathrm{~m} / \mathrm{s}^{2}$. This gives a typical range of $h_{1} / a=0.05$ to $0.2, D / a^{4}=0.569$ to $36.429, K / a=0.045$ to 0.18 , which are similar to those in Sturova [15] for the fully linear wave radiation problem.

Fig. 2 gives the root of the above equation $k_{1} a$ against $v a$ with different ice sheet thicknesses $h_{1} / a=0.05,0.1$ and 0.2 , together with the free surface case in which $h_{1}=0, D / a^{4}=0$, $K / a=0$, and therefore $k_{1}=v$. The figure shows when $v a \ll 1, k_{1} \cong v$ in all cases. This can be explained by Eq. (59), in which the leading term in the denominator will be 1 when $v$ is small. Difference between curves increases when $v a$ increases. At very large $v$, it can be shown that $k_{1} \rightarrow \sqrt[4]{K v / D}$ if we rewrite Eq. (59) as $v=\left(D k_{1}^{4}+1\right) k_{1} /\left(K k_{1}+1\right)$. We can also see from the figure that $k_{1}$ decreases as the ice thickness $h_{1} / a$ increases. This indicates that the wavelength of the first flexural-wave component in the ice sheet in Eq. (38) is larger than that in the free surface case. On the other hand, the wave decays more slowly along the depth when there is an ice sheet.

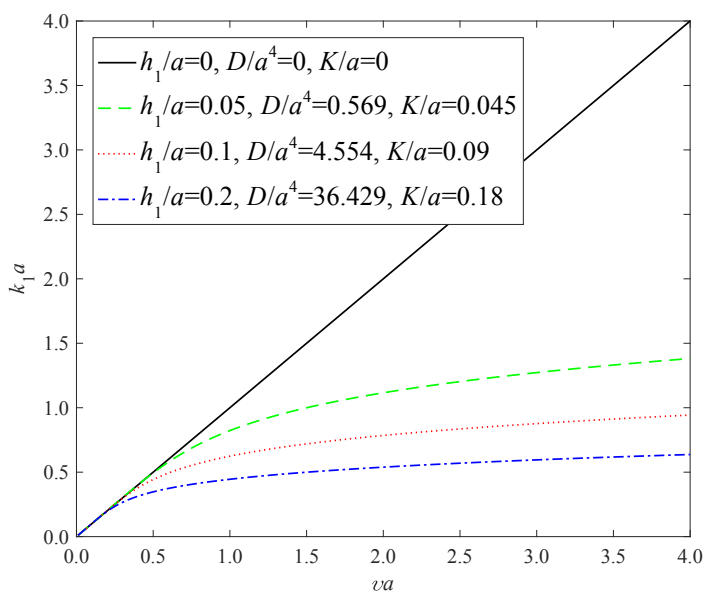

Fig. 2. Root of the dispersion Eq. (59) with different ice thicknesses.

\subsection{Convergence study and comparison}

To conduct the convergence study with $M$ and $S$, we consider the case of the cylinder undergoing clockwise circular oscillations beneath the ice sheet. A relatively large amplitude $\eta / a=0.8$ is taken to ensure the choices $M$ and $S$ are also suitable for other more mild amplitudes. The other parameters are $v a=2, h / a=2, h_{1} / a=0.15, D / a^{4}=15.368$, $K / a=0.135$.

The horizontal and vertical forces with different $M$ and $S$ are shown in Fig. 3, while the time history of the deformation of the ice sheet at $x=0$ as well as the wave profile at $t / T=0.5$ are given in Fig. $4(a)$ and $(b)$ respectively. Here $T=2 \pi / \omega$ is the period of the oscillation. It can be seen that the results obtained with $M=5, S=10$ and with $M=8, S=20$ are graphically 
1 undistinguishable. This means that the results have converged, and $M=5$ and $S=10$ will be 2 used in the following simulations, unless otherwise specified. The horizontal steady force computed by taking the average of the force over a period and by using the far field formula is also provided. Fig. 3 (a) shows that they are in excellent agreement.

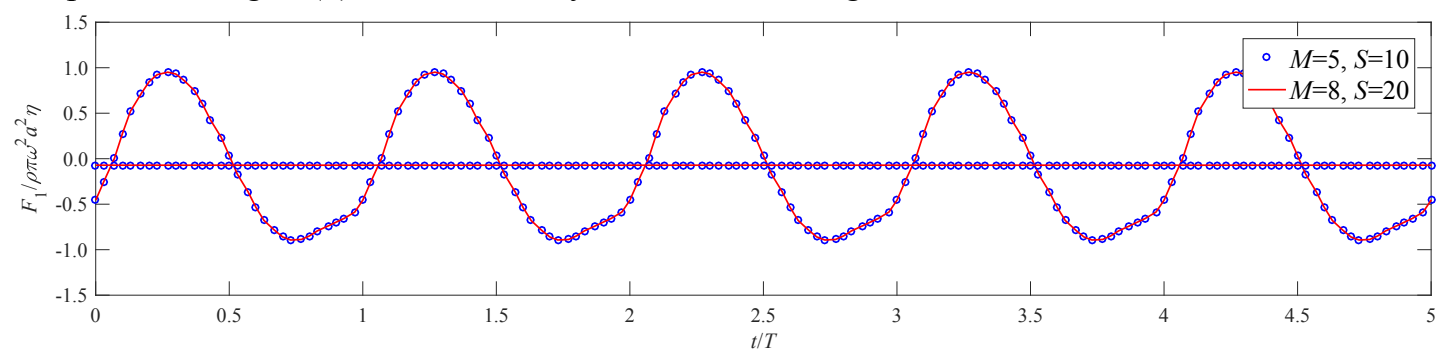

5

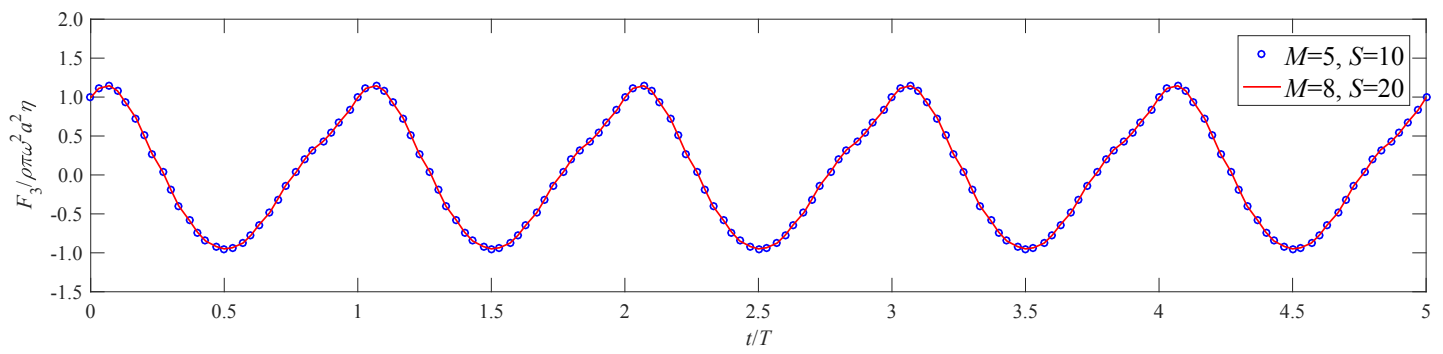

6

7

8

9

10

11

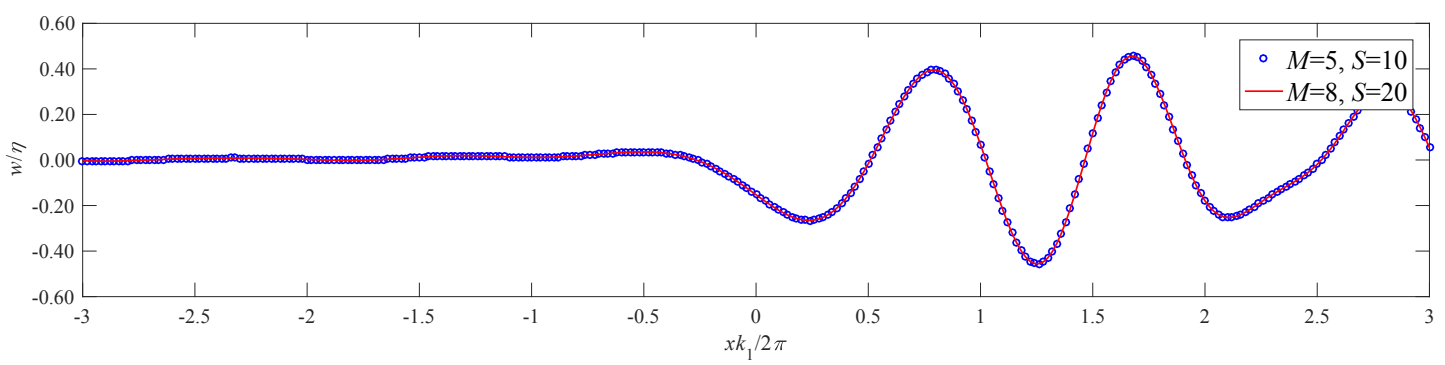

12

Fig. 3. Hydrodynamic force with different $M$ and $S$. (a) horizontal force; $(b)$ vertical force. $(\eta / a=0.8$; $\left.v a=2 ; h / a=2 ; h_{1} / a=0.15, D / a^{4}=15.368, K / a=0.135\right)$. The horizontal line in $(a)$ is the steady force which is computed by taking $M=5$ and $S=10$ : open circles: by taking the average of the force over a period; solid line: by using the far field formula.

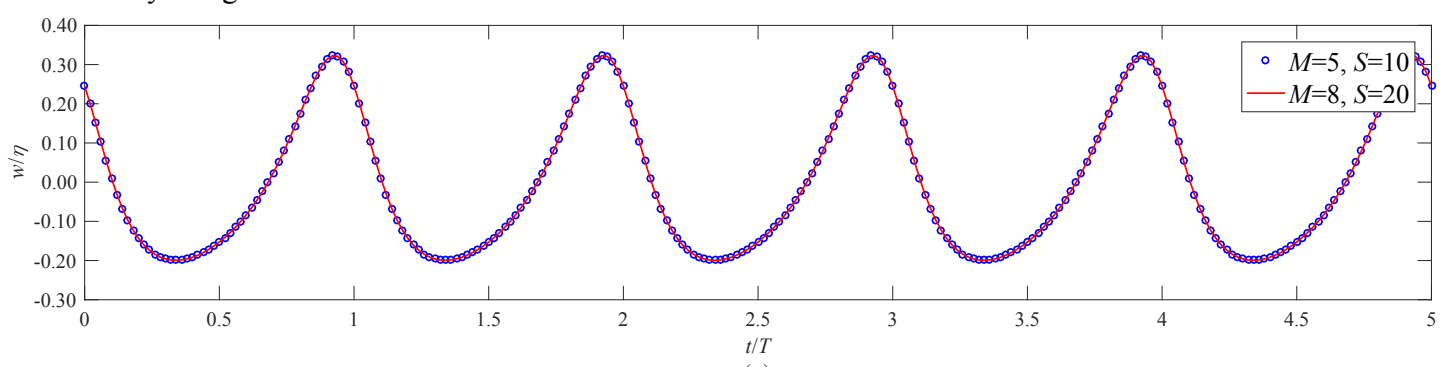

(a)

(b)

Fig. 4. Deformation of the ice sheet with different $M$ and $S$. (a) time history of the deformation at $x=0 ;(b)$ profile of the deformation at $t / T=0.5$. $\left(\eta / a=0.8 ; v a=2 ; h / a=2 ; h_{1} / a=0.15, D / a^{4}=15.368\right.$, $K / a=0.135)$ 


\subsection{Purely horizontal motion}

In this and the following sections, the wave radiation problems by a submerged horizontal circular cylinder undergoing various forced sinusoidal oscillations in water with an ice cover is studied.

The influence of oscillation amplitude, frequency and ice thickness on the nonlinear features of the hydrodynamic force and deformation of the ice sheet are analyzed.

We first consider the case of purely horizontal motions. Fig. 5 and Fig. 6 respectively show the horizontal and vertical forces at different motion amplitudes $\eta_{1} / a=0.1,0.4$ and 0.8 respectively.

The other parameters are $v a=0.5, h / a=2$ and $h_{1} / a=0.15, D / a^{4}=15.368, K / a=0.135$. The fully linear solutions are also provided. We notice that for the horizontal force, the nonlinear effect is small. This is can be partly explained by the fact that when the body is in such a motion, the horizontal force only consists of components of frequencies $(2 n+1) \omega$, while the vertical force consists of components of frequencies $2 n \omega, n=0,1,2, \ldots,[20]$. The horizontal force is dominated by the component of $\omega$, which is almost linearly proportional to $\eta_{1}$. Thus the nonlinear effect in Fig. 5 is not obvious. For the same reason, we notice that the vertical force in Fig. 6 is dominated by the $2 \omega$ terms and the nonlinear effect is evident, i.e. the amplitudes of both the steady component and the higher components increase with the oscillation amplitude. However, the vertical force is zero for the fully linear solution.

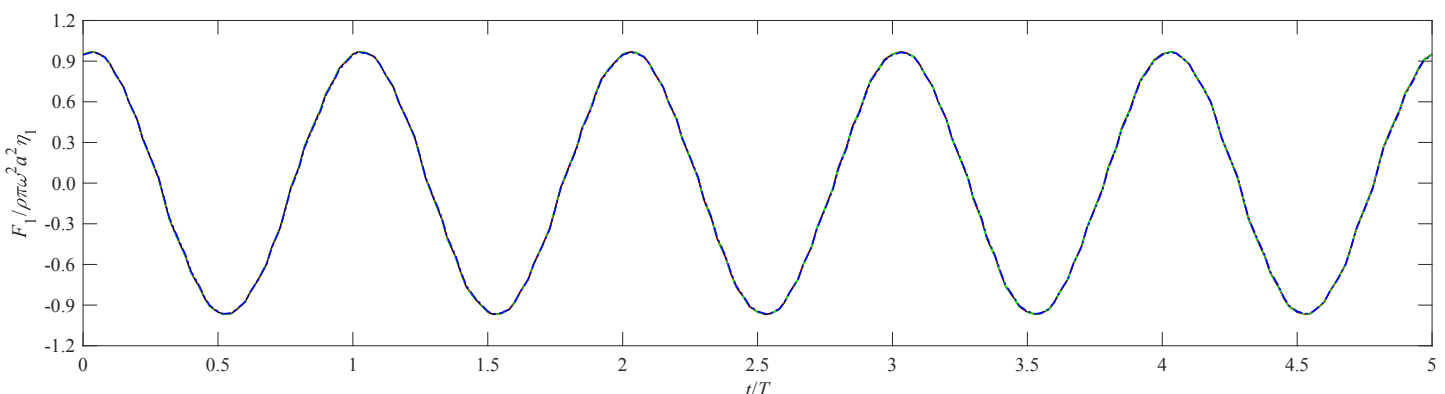

Fig. 5. Horizontal force at different $\eta_{1}\left(\eta_{3}=0\right)$ : solid line: fully linear solution; dashed line: $\eta_{1} / a=0.1$; dotted line: $\eta_{1} / a=0.4$; dash-dotted line: $\eta_{1} / a=0.8$. ( $v a=0.5 ; h / a=2 ; h_{1} / a=0.15$, $\left.D / a^{4}=15.368, K / a=0.135\right)$

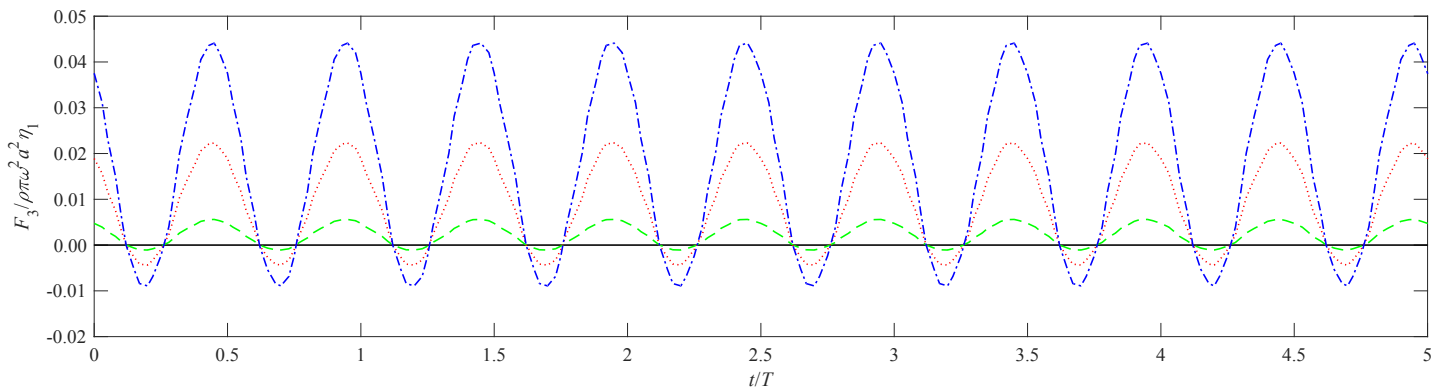

Fig. 6. Vertical force at different $\eta_{1}\left(\eta_{3}=0\right)$ : solid line: fully linear solution; dashed line: $\eta_{1} / a=0.1$; dotted line: $\eta_{1} / a=0.4$; dash-dotted line: $\eta_{1} / a=0.8$. $\left(v a=0.5 ; h / a=2 ; h_{1} / a=0.15, D / a^{4}=15.368\right.$, 
2 Fig. 7 gives the time history of the deformation of the ice sheet at $x=0$. For the fully linear case, the problem is antisymmetric about $x=0$, or $w(-x, t)=-w(-x, t)$, and therefore $w(0, t)=0$, as shown in the figure. For the nonlinear case we notice that the oscillation has a period $T / 2$, similar to the vertical force. Eqs. (27) and (44) show that in this case $D_{m}^{s}=(-1)^{s} C_{m}^{s}$. Substituting this and $x=0$ into Eq. (45) and noticing $q=u-s$, we find that only the terms of even $u$ have contributions to the deformation $w(0, t)$. Thus oscillation period in Fig. 7 is $T / 2$. This can also be explained based on the argument of $\mathrm{Wu}$ [21]. Because the body is symmetric about the vertical line passing its centre, during its periodic horizontal oscillation about $x=0, w(0, t)$ observed from one side (by looking into the page) is the $w(0, t+T / 2)$ observed from the other side (by looking out of the page), or $w(0, t)=w(0, t+T / 2)$. Such an argument is valid even when the fully nonlinear free surface boundary condition is used.

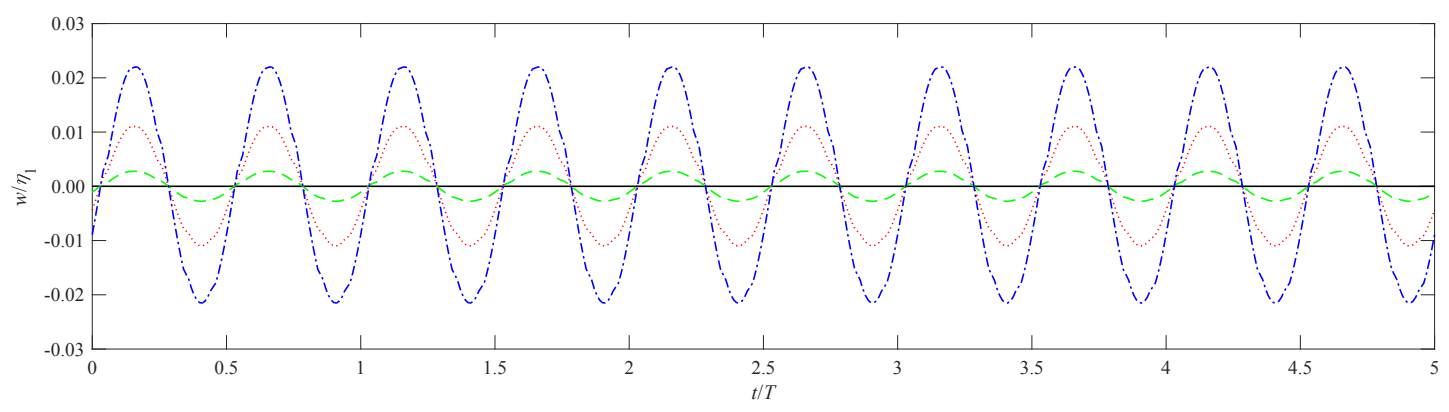

Fig. 7. Time history of the deformation at $x=0$ with different $\eta_{1}\left(\eta_{3}=0\right)$ : solid line: fully linear solution; dashed line: $\eta_{1} / a=0.1$; dotted line: $\eta_{1} / a=0.4$; dash-dotted line: $\eta_{1} / a=0.8$. ( $v a=0.5 ; h / a=2$; $h_{1} / a=0.15, D / a^{4}=15.368, K / a=0.135$

Fig. $8(a)$ shows the profiles of the deformation of the ice sheet with different $\eta_{1}$. When $\eta_{1}$ is small, the profile of the deformation is proportional to $\eta_{1}$ linearly. When $\eta_{1}$ increases, the higher components appear and the change of the profiles becomes noticeable. The profile becomes asymmetric about $x=0$. In fact substituting $D_{m}^{s}=(-1)^{s} C_{m}^{s}$ into Eq. (45), we can find that the components of the profile with an even $u$ is symmetric about $x=0$, while the components with an odd $u$ is anti-symmetric about $x=0$. Profiles obtained from Eq.(64) and far field formula in Eq. (38) are presented in Fig. $8(b)$. We can see that the local wave in Eq. (37) attenuates quite fast, and the wave tends to that obtained from Eq. (38) quickly. In fact, when $\left|x k_{1} / 2 \pi\right|>1.0$ there is hardly any graphical difference between the two curves. 


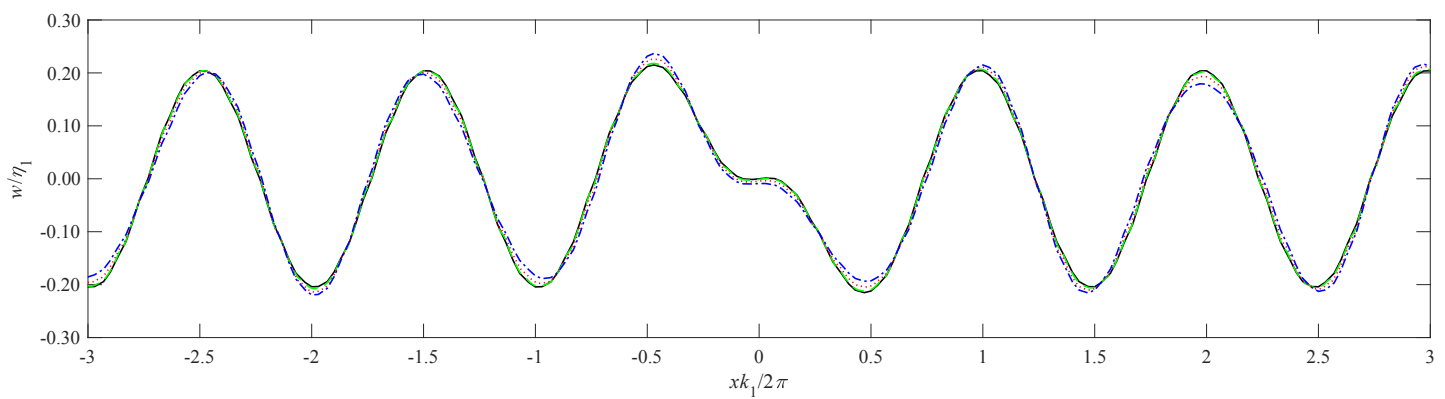

1

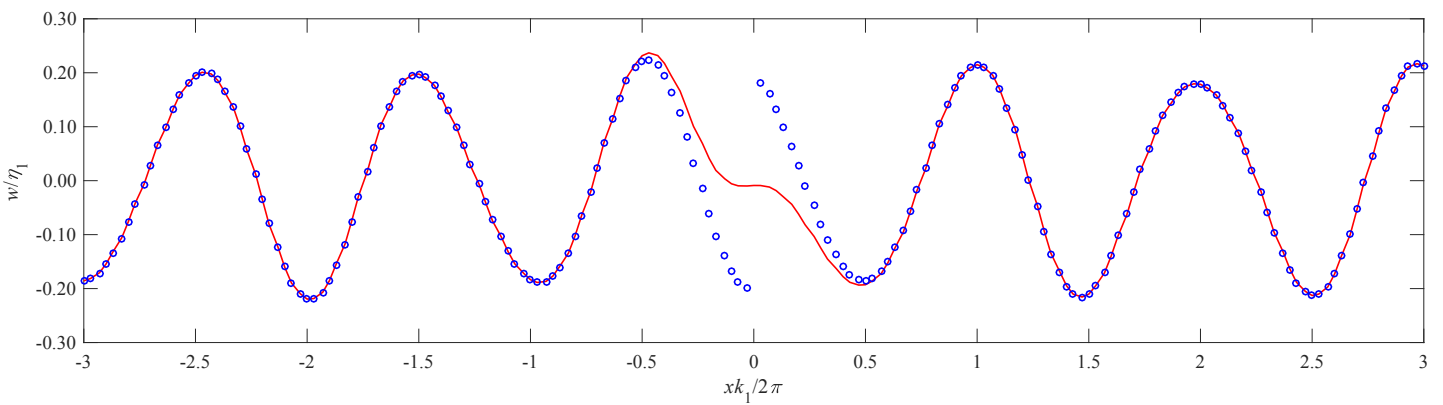

(b)
2

3

Fig. 8. Profile of the deformation at $t / T=0.5$. (a) profiles with different $\eta_{1}\left(\eta_{3}=0\right)$ : solid line: fully linear solution; dashed line: $\eta_{1} / a=0.1$; dotted line: $\eta_{1} / a=0.4$; dash-dotted line: $\eta_{1} / a=0.8$; $(b)$ profiles at $\eta_{1} / a=0.8\left(\eta_{3}=0\right)$ : solid line: Eq. (37); open circles: far field formula Eq. (38). (va=0.5; $h / a=2$; $h_{1} / a=0.15, D / a^{4}=15.368, K / a=0.135$ )

Then the hydrodynamic force and deformation of the ice sheet at different oscillation frequencies $v a=0.1,1,2,4$ are computed. Other parameters are taken as $\eta_{1} / a=0.8, h / a=2, h_{1} / a=0.15$, $D / a^{4}=15.368$ and $K / a=0.135$. It can be seen from Fig. 9 that the amplitude of the horizontal force which has components at $(2 n+1) \omega$ only decreases when $v$ increases, and tends to a constant value very quickly. The vertical force which has components at $2 n \omega$ only tends to a constant more slowly when $v$ increases, as shown in Fig. 10. The leading term in the horizontal force is $\omega$, which is dominated by the linear contribution, while in the vertical force, the leading term is from the nonlinear contribution. From Figs. 9 and 10, it can be seen that the nonlinear contribution is more sensitive to the variation of $v$. The time history of the ice sheet deformation at $x=0$ and its profile at $t / T=0.5$ are respectively given in Fig. 11 and Fig. 12 and these results are highly frequency dependent. It can be seen when $v$ is small, the deformation shape is very erratic, which shows that various components are highly significant.

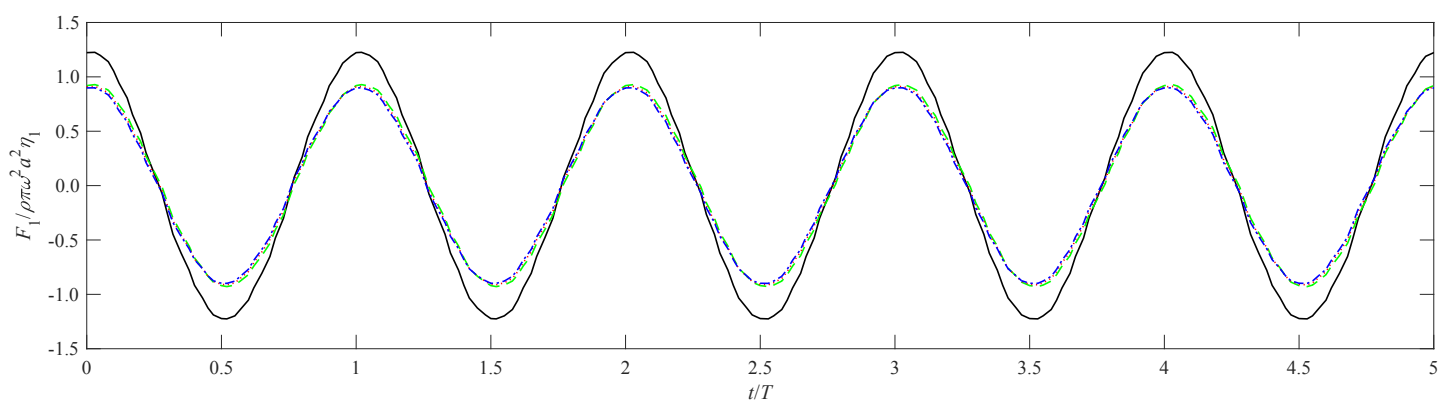



dotted line: $v a=4 .\left(\eta_{1} / a=0.8 ; h / a=2 ; h_{1} / a=0.15, D / a^{4}=15.368, K / a=0.135\right)$

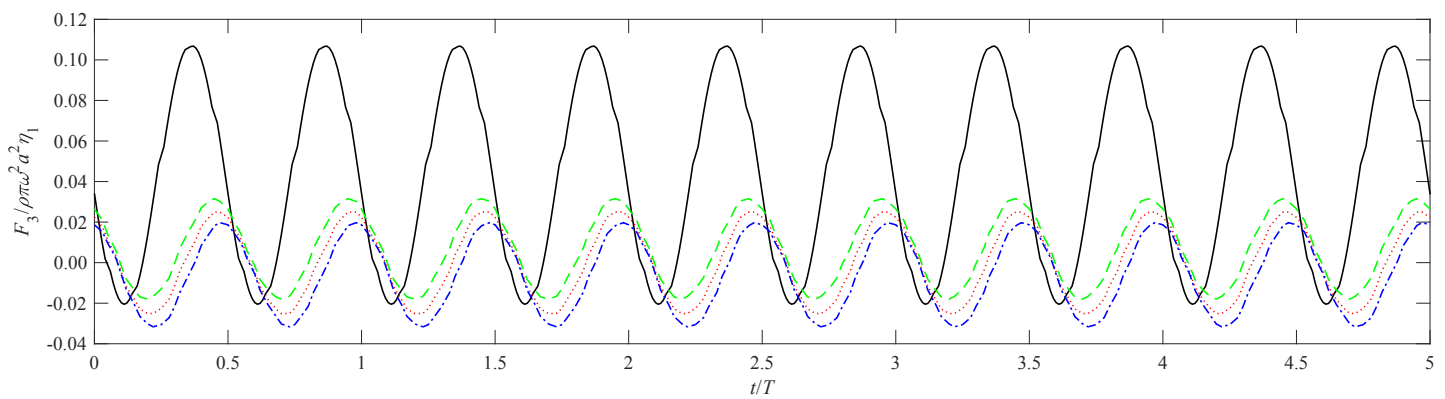

Fig. 10. Vertical force at different $v$ : solid line: $v a=0.1$; dashed line: $v a=1$; dotted line: $v a=2$; dashdotted line: $v a=4 .\left(\eta_{1} / a=0.8 ; h / a=2 ; h_{1} / a=0.15, D / a^{4}=15.368, K / a=0.135\right)$

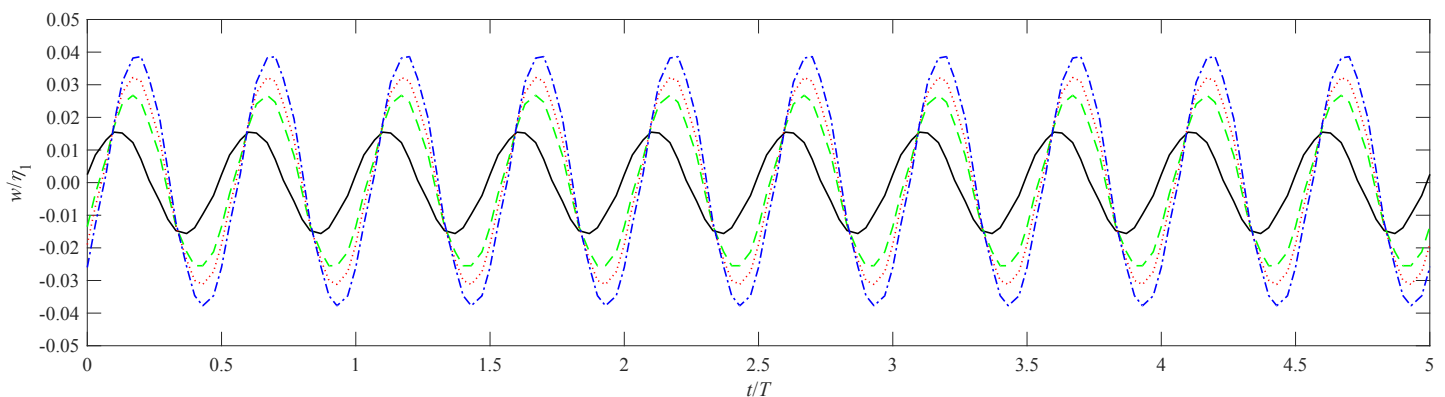

Fig. 11. Time history of the deformation at $x=0$ with different $v$ : solid line: $v a=0.1$; dashed line: $v a=1$; dotted line: $v a=2$; dash-dotted line: $v a=4 .\left(\eta_{1} / a=0.8 ; h / a=2 ; h_{1} / a=0.15, D / a^{4}=15.368\right.$, $K / a=0.135)$

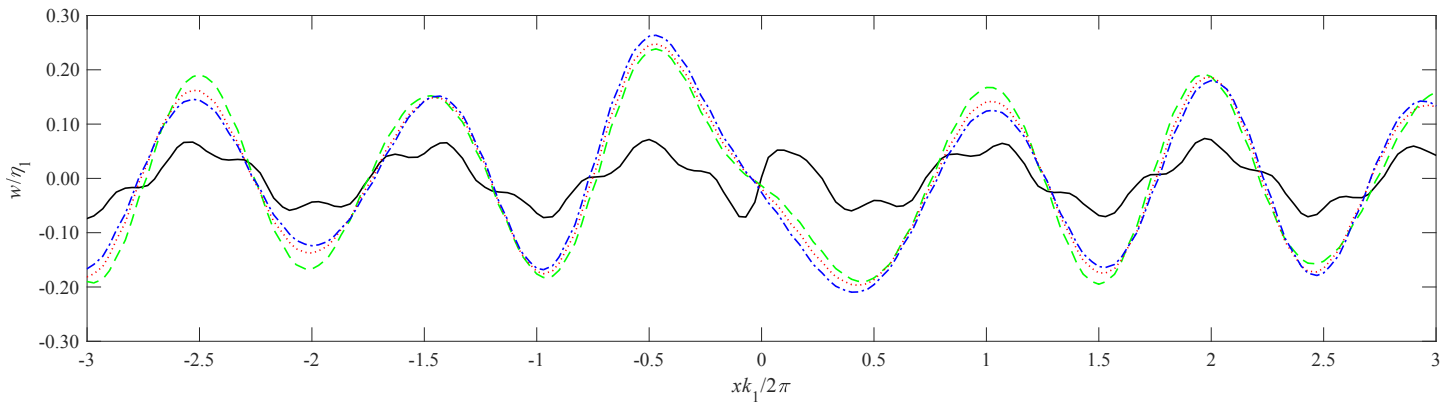

Fig. 12. Profile of the deformation at $t / T=0.5$ with different $v$ : solid line: $v a=0.1$; dashed line: $v a=1$; dotted line: $v a=2$; dash-dotted line: $v a=4 .\left(\eta_{1} / a=0.8 ; h / a=2 ; h_{1} / a=0.15, D / a^{4}=15.368\right.$, $K / a=0.135)$

We undertake further simulations to investigate the effect of ice thickness. $h_{1} / a$ is taken as 0.05 , $0.1,0.15$ and 0.2 respectively. The other parameters are respectively chosen as $\eta_{1} / a=0.8$, $h / a=2$ and $v a=3$. It can be seen from Fig. 13 that the effect of the thickness on the horizontal force is small at this frequency. However, its effect is highly significant on the vertical force, for example the steady component increases with $h_{1}$, as shown in Fig. 14. Fig. 15 and Fig. 16 respectively show the time history and profile of the deformation of the ice sheet. We notice that 
1 as $h_{1}$ increases, the ice sheet becomes more rigid and therefore its deformation becomes smaller 2 as shown in the figures. Fig. 15 shows that the peaks and troughs at different $h_{1} / a$ do not occur at 3 the same $t$, which implies that the higher oscillatory components in the wave are significant. Fig. 416 shows that the nonlinear effect of the body motion on the deformation of the ice sheet is most significant right above the body, and the results from different $h_{1}$ are much closer away from the body.

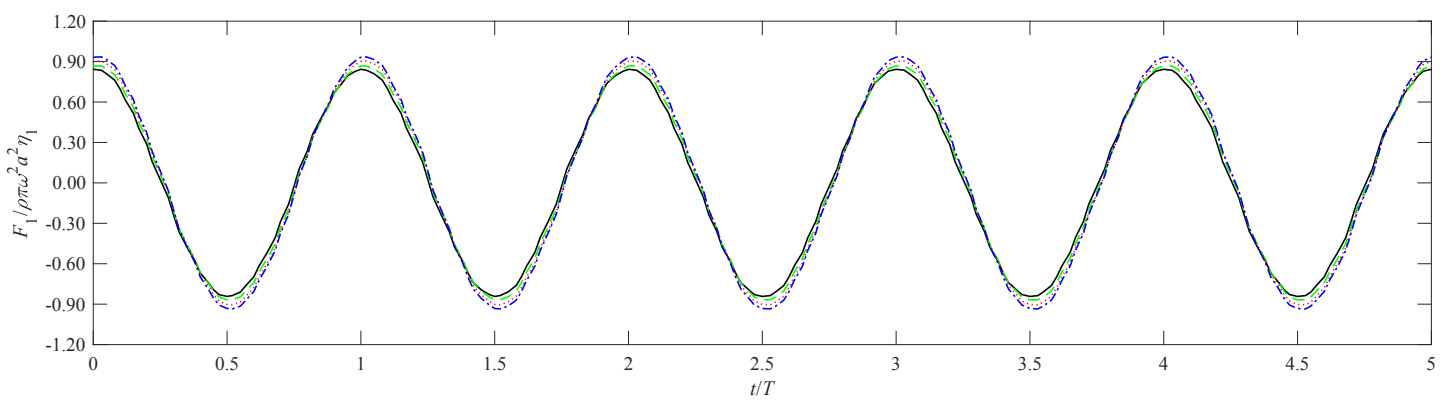

Fig. 13. Horizontal force at different $h_{1}$ : solid line: $h_{1} / a=0.05, D / a^{4}=0.569, K / a=0.045$; dashed line: $\quad h_{1} / a=0.1, \quad D / a^{4}=4.554, \quad K / a=0.09 ;$ dotted line: $h_{1} / a=0.15, D / a^{4}=15.368$, $K / a=0.135$; dash-dotted line: $h_{1} / a=0.2, D / a^{4}=36.429, K / a=0.18$. $\left(\eta_{1} / a=0.8 ; v a=3\right.$; $h / a=2)$

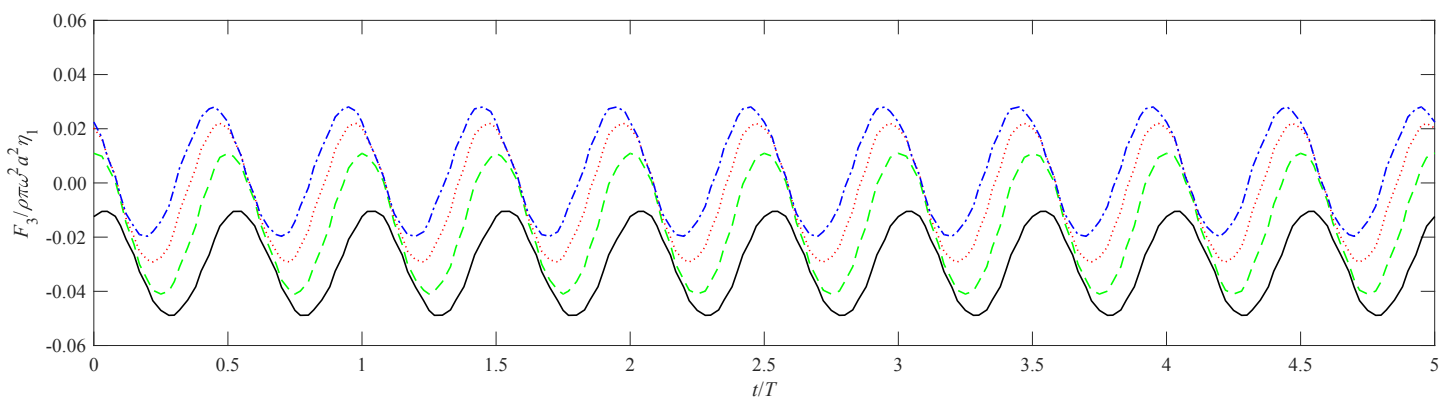

Fig. 14. Vertical force at different $h_{1}$ : solid line: $h_{1} / a=0.05, D / a^{4}=0.569, K / a=0.045$; dashed line: $h_{1} / a=0.1, D / a^{4}=4.554, K / a=0.09$; dotted line: $h_{1} / a=0.15, D / a^{4}=15.368, K / a=0.135$; dash-dotted line: $h_{1} / a=0.2, D / a^{4}=36.429, K / a=0.18 .\left(\eta_{1} / a=0.8 ; v a=3 ; h / a=2\right)$

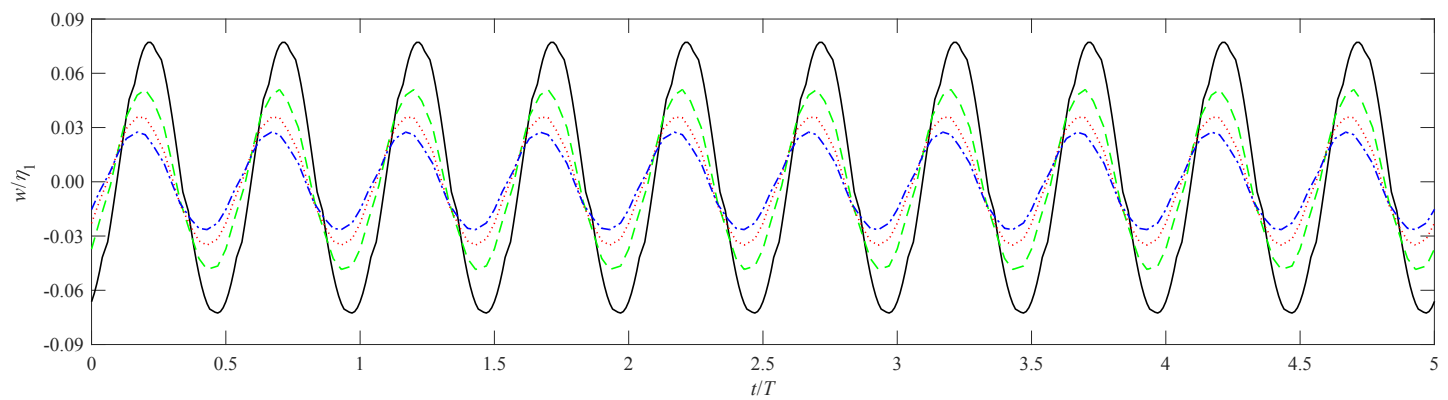

Fig. 15. Time history of the deformation at $x=0$ with different $h_{1}$ : solid line: $h_{1} / a=0.05, D / a^{4}=0.569$, $K / a=0.045$; dashed line: $h_{1} / a=0.1, D / a^{4}=4.554, K / a=0.09$; dotted line: $h_{1} / a=0.15$, $D / a^{4}=15.368, \quad K / a=0.135 ; \quad$ dash-dotted line: $h_{1} / a=0.2, \quad D / a^{4}=36.429, \quad K / a=0.18$ （ 


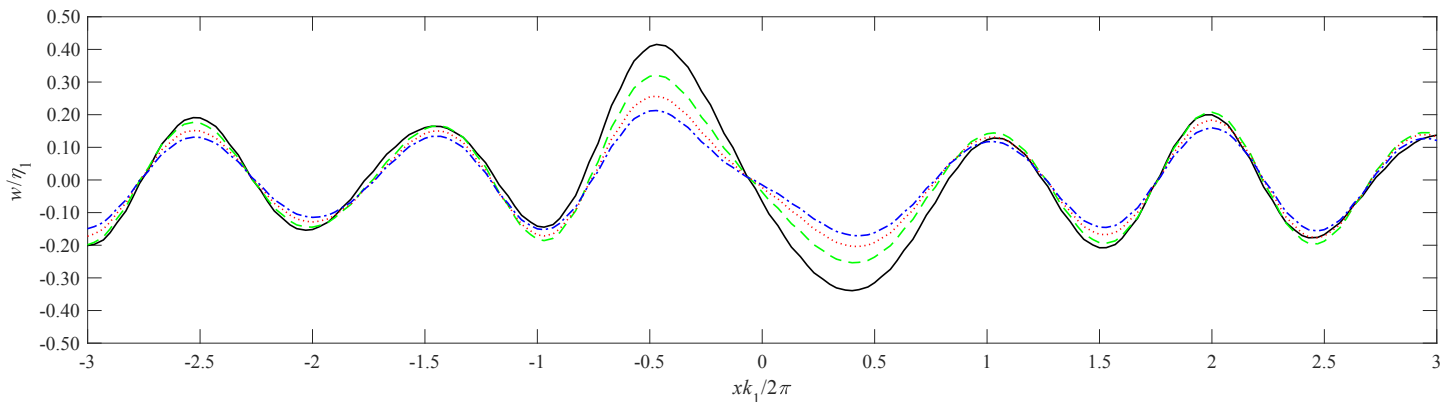

Fig. 16. Deformation of the ice sheet at $t / T=0.5$ with different $h_{1}$ : solid line: $h_{1} / a=0.05, D / a^{4}=0.569$, $K / a=0.045$; dashed line: $h_{1} / a=0.1, D / a^{4}=4.554, K / a=0.09$; dotted line: $h_{1} / a=0.15$, $D / a^{4}=15.368, K / a=0.135 ;$ dash-dotted line: $h_{1} / a=0.2, D / a^{4}=36.429, K / a=0.18$. $\left.\eta_{1} / a=0.8 ; v a=3 ; h / a=2\right)$

\subsection{Purely vertical motion}

Simulations are undertaken at $v a=0.5, \quad h / a=2$ and $h_{1} / a=0.15, \quad D / a^{4}=15.368$, $K / a=0.135$ for $\eta_{3} / a=0.1,0.4$ and 0.8 respectively, together with the fully linear solutions. In this case, the horizontal force is zero due to symmetry. The time histories of the vertical force is presented in Fig. 17, and the corresponding deformation of the ice sheet at $x=0$ is shown in Fig. 18. The obtained results are normalized by $\eta_{3}$. Thus when $\eta_{3}$ is small, the curves should coincide with that obtained by the fully linear solutions, as shown in these two figures. When $\eta_{3}$ increases, the nonlinear effect appears. We can see that the nonlinearity is particularly strong at the crest of the force. Similar nonlinear features are also observed in the deformations in Fig. 19 (a). The deformations respectively computed by the far field formula Eq. (38) and by Eq. (37) are compared in Fig. 19 (b). It can be seen that as in the previous case the local wave attenuates fast, and when $\left|x k_{1} / 2 \pi\right|>1.0$ these two curves nearly coincide with each other.

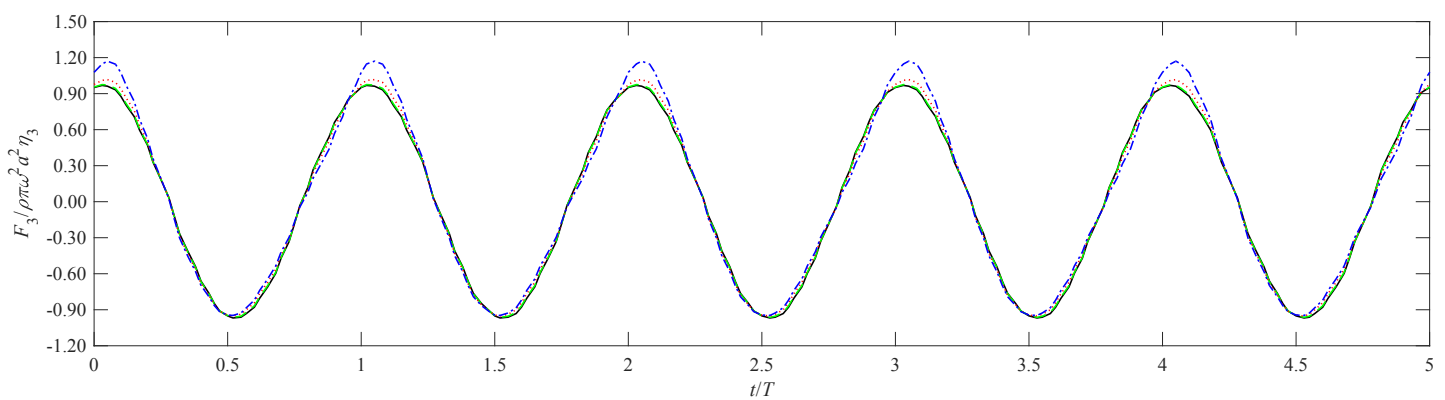

Fig. 17. Vertical force at different $\eta_{3}\left(\eta_{1}=0\right)$ : solid line: fully linear solution; dashed line: $\eta_{3} / a=0.1$; dotted line: $\eta_{3} / a=0.4$; dash-dotted line: $\eta_{3} / a=0.8$. $\left(v a=0.5 ; h / a=2 ; h_{1} / a=0.15, D / a^{4}=15.368\right.$, $K / a=0.135)$ 


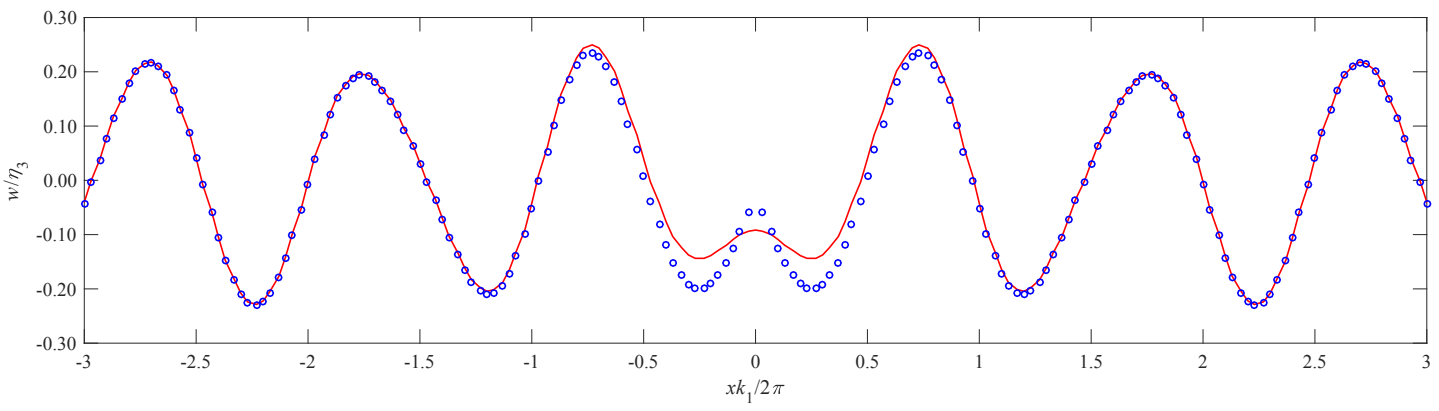

Fig. 18. Time history of the deformation at $x=0$ with different $\eta_{3}\left(\eta_{1}=0\right)$ : solid line: fully linear solution; dashed line: $\eta_{3} / a=0.1$; dotted line: $\eta_{3} / a=0.4$; dash-dotted line: $\eta_{3} / a=0.8$. (va=0.5; $h / a=2$; $\left.h_{1} / a=0.15, D / a^{4}=15.368, K / a=0.135\right)$

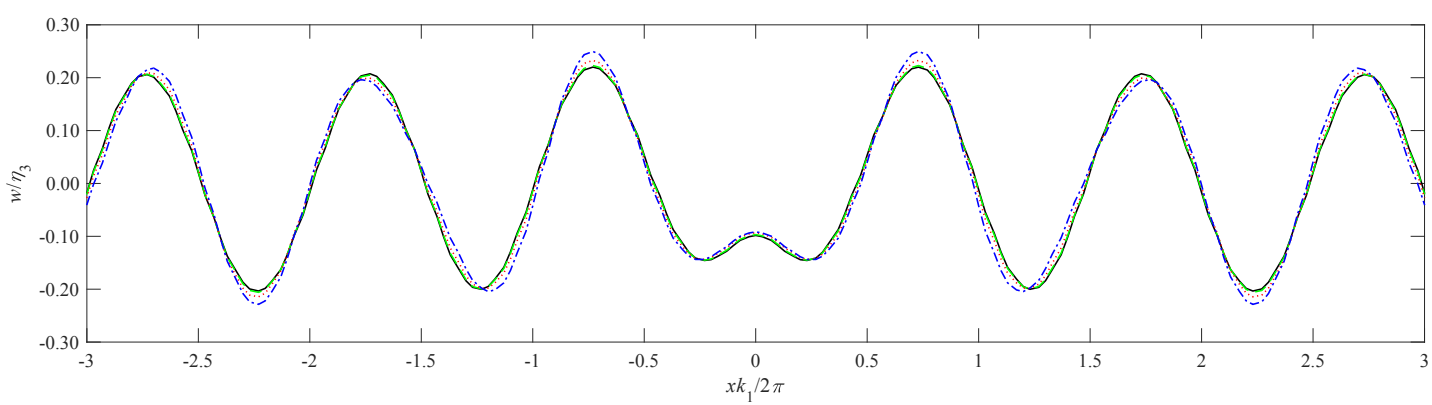

(a)
6

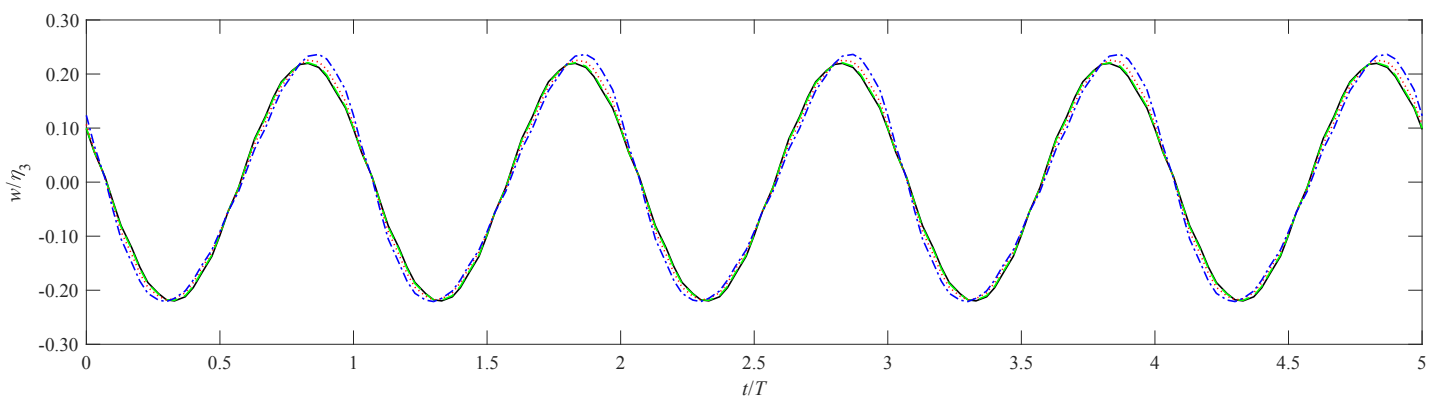

(b)

Fig. 19. Profile of the deformation at $t / T=0.5$. (a) profiles with different $\eta_{3}\left(\eta_{1}=0\right)$ : solid line: fully linear solution; dashed line: $\eta_{3} / a=0.1$; dotted line: $\eta_{3} / a=0.4$; dash-dotted line: $\eta_{3} / a=0.8 ;(b)$ profiles at $\eta_{3} / a=0.8\left(\eta_{1}=0\right)$ : solid line: Eq. (37); open circles: far field formula Eq. (38). ( $v a=0.5 ; h / a=2$; $\left.h_{1} / a=0.15, D / a^{4}=15.368, K / a=0.135\right)$

Then the computations are carried out at different oscillation frequencies, with $v a=0.1,1,2$, and 4. The other parameters are taken as $\eta_{3} / a=0.8, h / a=2$ and $h_{1} / a=0.15, D / a^{4}=15.368$, $K / a=0.135$. The time histories of the vertical force with different $v$ are shown in Fig. 20. We can see that the vertical force decreases fast when $v$ increases. As shown in Section 4.1, when $v$ increases, the variation of wave number $k_{1}$ with ice sheet is much milder than that with free surface, especially at large $v$. As $k_{1}$ has major effect on the result, the variation of $v$ on the vertical force becomes small when $v a>1$, as shown in Fig. 20. Similar behavior can be seen in the deformation presented in Fig. 21 and Fig. 22. Compared with the purely horizontal motion, it is important to note the distance from the centre of the cylinder to ice sheet changes during the 


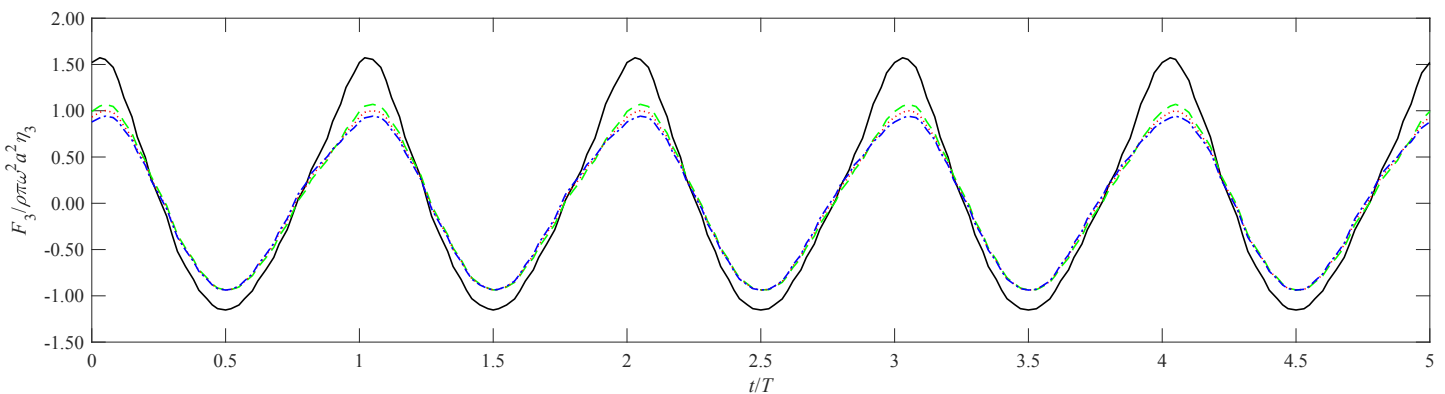

Fig. 20. Vertical force at different $v$ : solid line: $v a=0.1$; dashed line: $v a=1$; dotted line: $v a=2$; dashdotted line: $v a=4 .\left(\eta_{3} / a=0.8 ; h / a=2 ; h_{1} / a=0.15, D / a^{4}=15.368, K / a=0.135\right)$

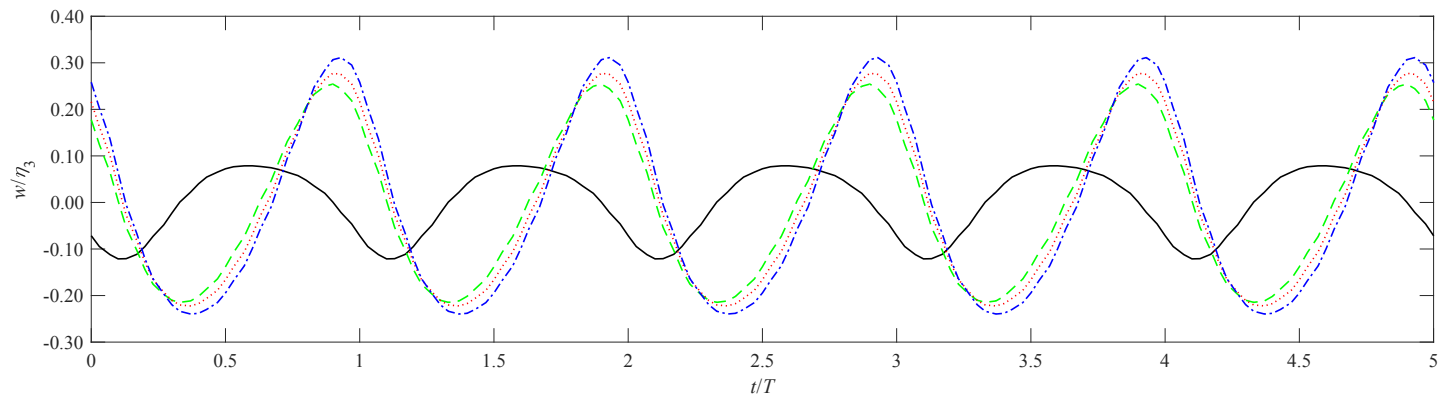

Fig. 21. Time history of the deformation at $x=0$ with different $v$ : solid line: $v a=0.1$; dashed line: $v a=1$; dotted line: $v a=2$; dash-dotted line: $v a=4 .\left(\eta_{3} / a=0.8 ; h / a=2 ; h_{1} / a=0.15, D / a^{4}=15.368\right.$, $K / a=0.135$

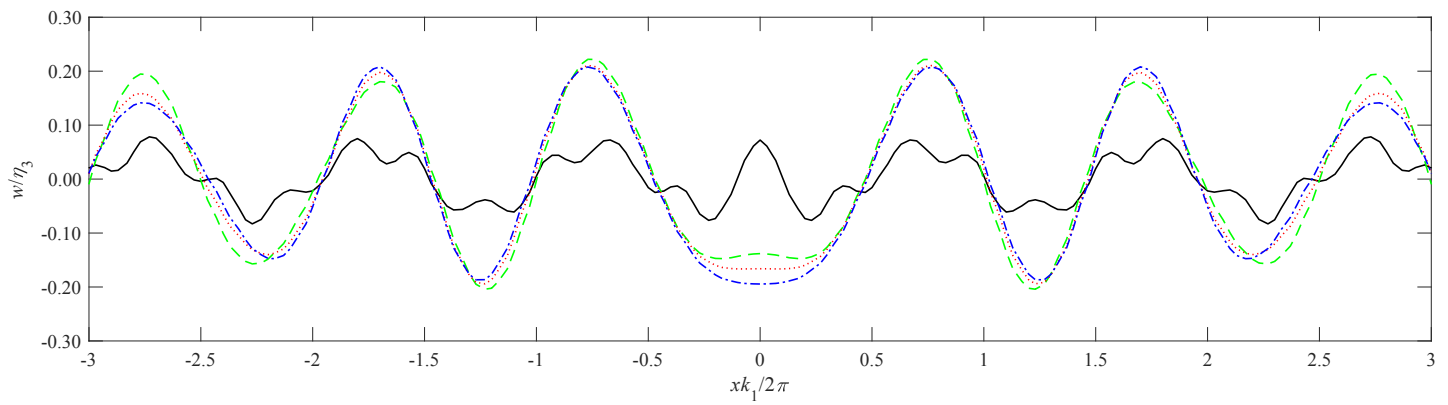

Fig. 22. Profile of the deformation at $t / T=0.5$ with different $v$ : solid line: $v a=0.1$; dashed line: $v a=1$; dotted line: $v a=2$; dash-dotted line: $v a=4 .\left(\eta_{3} / a=0.8 ; h / a=2 ; h_{1} / a=0.15\right.$, $\left.D / a^{4}=15.368, K / a=0.135\right)$

Simulations are further carried out at $h_{1} / a=0.05,0.1,0.15$ and 0.2 , with $\eta_{3} / a=0.8, h / a=2$ and $v a=3$. The time histories of the vertical force are shown in Fig. 23. It can be seen that $h_{1}$ has a strong effect on the crest of the vertical force, while its effect on the trough is much smaller. We notice in Figs. 24 and 25 that smaller $h_{1}$ leads to a larger deformation, due to the fact that the ice is more flexible. As a result, vertical motion of the cylinder generates force of stronger nonlinearity 


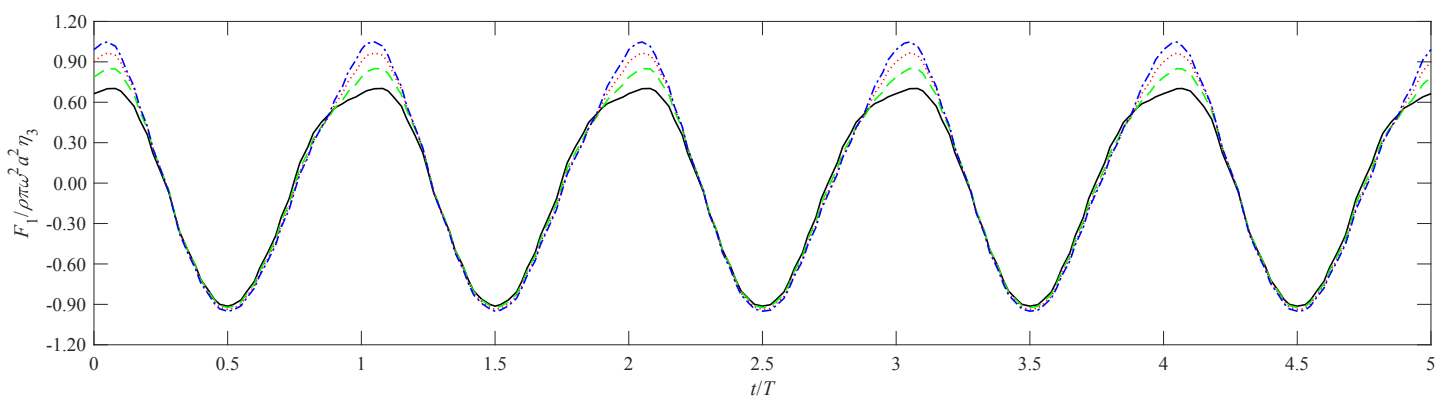

2 Fig. 23. Vertical force at different $h_{1}$ : solid line: $h_{1} / a=0.05, D / a^{4}=0.569, K / a=0.045$; dashed line: $h_{1} / a=0.1, D / a^{4}=4.554, K / a=0.09$; dotted line: $h_{1} / a=0.15, D / a^{4}=15.368, K / a=0.135$; dash-dotted line: $h_{1} / a=0.2, D / a^{4}=36.429, K / a=0.18 .\left(\eta_{3} / a=0.8 ; v a=3 ; h / a=2\right)$

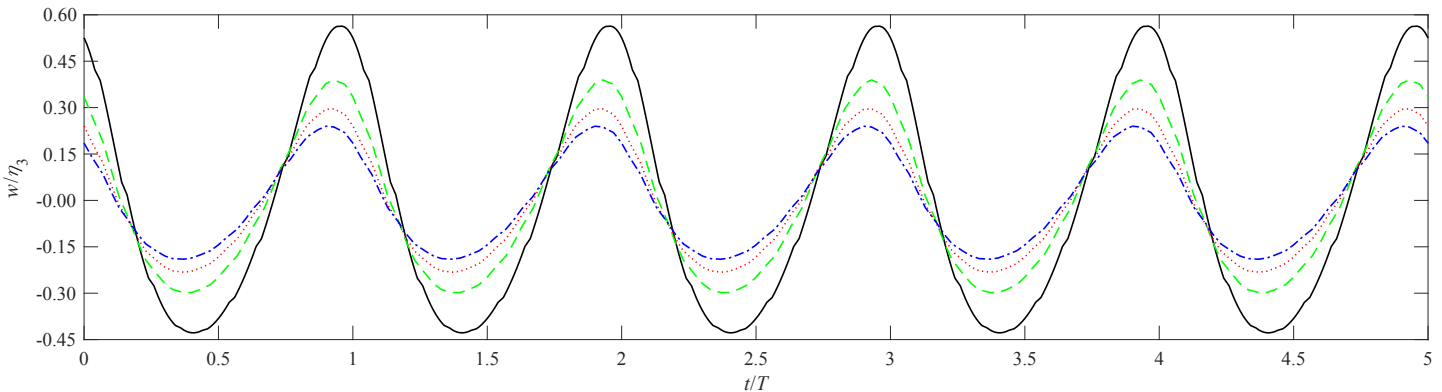

Fig. 24. Time history of the deformation at $x=0$ with different $h_{1}$ : solid line: $h_{1} / a=0.05, D / a^{4}=0.569$, $K / a=0.045$; dashed line: $h_{1} / a=0.1, D / a^{4}=4.554, K / a=0.09$; dotted line: $h_{1} / a=0.15$, $D / a^{4}=15.368, K / a=0.135 ;$ dash-dotted line: $h_{1} / a=0.2, \quad D / a^{4}=36.429, \quad K / a=0.18$. $\left.\eta_{3} / a=0.8 ; v a=3 ; h / a=2\right)$

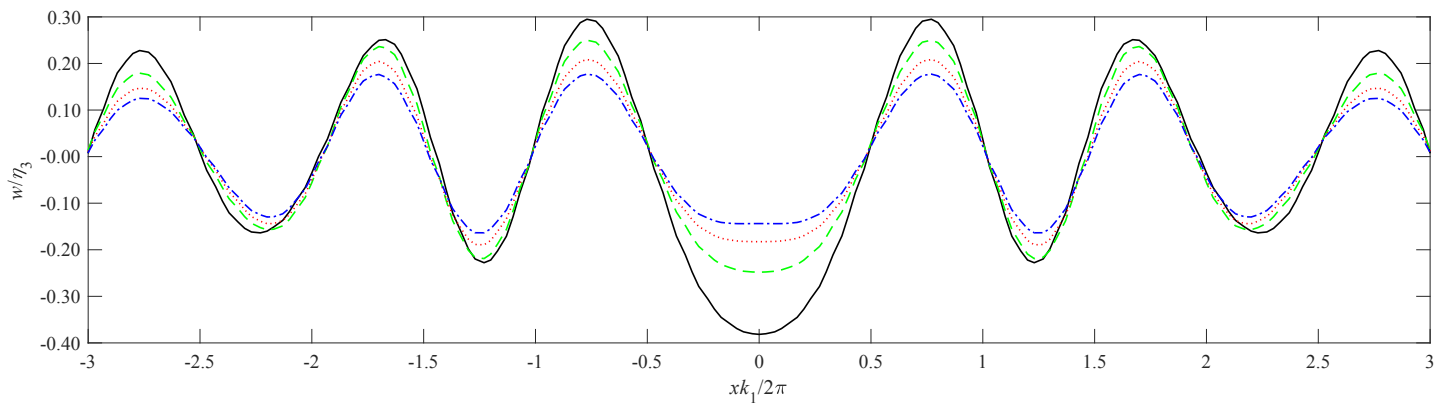

Fig. 25. Deformation of the ice sheet at $t / T=0.5$ with different $h_{1}$ : solid line: $h_{1} / a=0.05, D / a^{4}=0.569$, $K / a=0.045$; dashed line: $h_{1} / a=0.1, D / a^{4}=4.554, K / a=0.09$; dotted line: $h_{1} / a=0.15$, $D / a^{4}=15.368, K / a=0.135$; dash-dotted line: $h_{1} / a=0.2, D / a^{4}=36.429, K / a=0.18$. ( $\left.\eta_{3} / a=0.8 ; v a=3 ; h / a=2\right)$

\subsection{Circular motion}

The hydrodynamic forces along $x$ and $y$ directions are respectively presented in Fig. 26 and Fig. 27 for clockwise motion with three different amplitudes, at $v a=0.5, h / a=2$ and $h_{1} / a=0.15$, $D / a^{4}=15.368, K / a=0.135 . \eta / a$ is set to be $0.1,0.4$ and 0.8 respectively. The fully linear 
solutions are also provided for a comparison. As expected, the nonlinearity becomes important at two larger amplitudes. At $\eta / a=0.8$, near the trough of the horizontal force, the curve becomes nearly flat, when the peak of the vertical force becomes sharper.

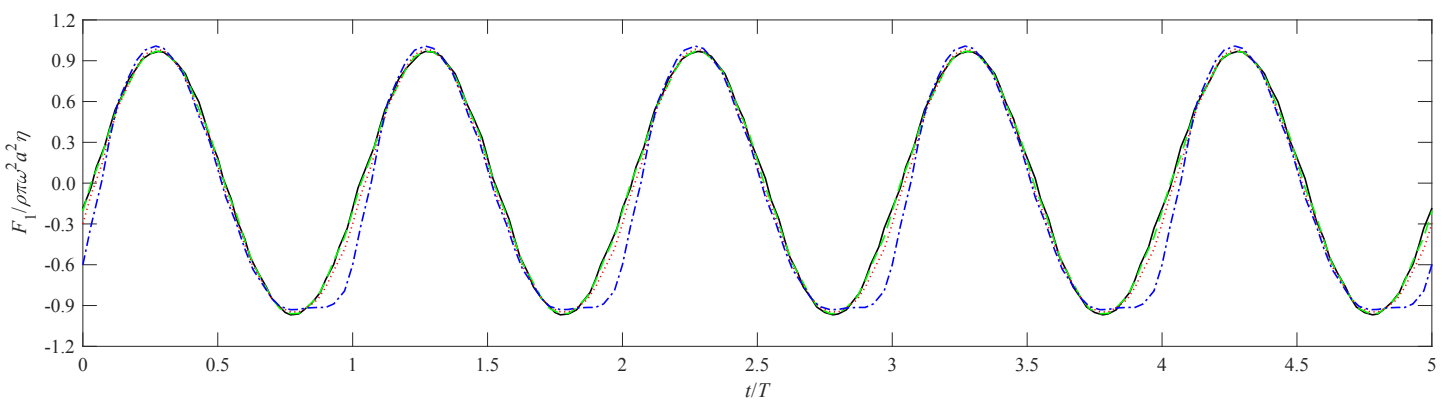

Fig. 26. Horizontal force at different $\eta$ : solid line: fully linear solution; dashed line: $\eta / a=0.1$; dotted line: $\eta / a=0.4 ;$ dash-dotted line: $\eta / a=0.8 . \quad\left(v a=0.5 ; h / a=2 ; h_{1} / a=0.15, D / a^{4}=15.368\right.$, $K / a=0.135)$

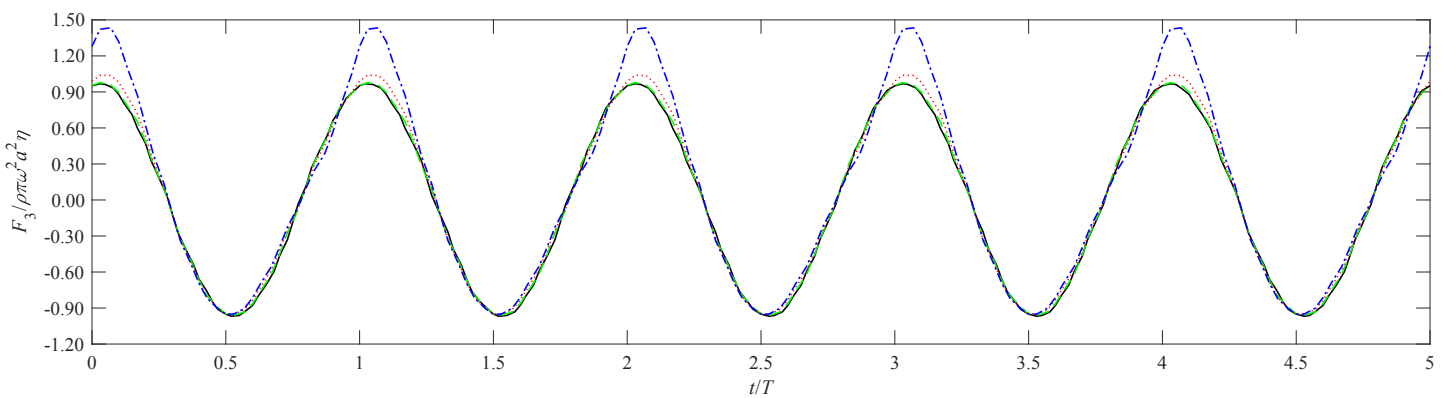

Fig. 27. Vertical force at different $\eta$ : solid line: fully linear solution; dashed line: $\eta / a=0.1$; dotted line: $\eta / a=0.4 ;$ dash-dotted line: $\eta / a=0.8 . \quad\left(v a=0.5 ; h / a=2 ; h_{1} / a=0.15, D / a^{4}=15.368\right.$, $K / a=0.135)$

Fig. 28 shows the corresponding time histories of the deformation at $x=0$ with different $\eta$. We can see that the effect of variation of $\eta$ in circular motion is stronger than that in horizontal or vertical motion. The profiles of deformation of the ice sheet at $t / T=0.5$ are shown in Fig. $29(a)$. It is interesting to see from the figure that the wave propagates mainly to the right hand side, and there is hardly any wave propagating to the left. In fact, it is known that based on the fully linear theory for the free surface flow problem, when a circular cylinder is in clockwise motion, no wave will propagate to $x=-\infty$ [22]. This conclusion can be found to be also valid for the present ice cover case. In fact this can be confirmed in Eq. (57) by taking $p=0, s=1$. However, when the fully nonlinear boundary condition is imposed on the body surface, Eq. (57) in section 3.5.4 shows that there will be wave at $x=-\infty$ although it may be small, which is the same as the free surface problem [18]. The wave at far field on the left hand side is shown in Fig. 30. It shows that when the nonlinear effect is included there will be wave at $x=-\infty$ even though it may be small. 
1

3

4

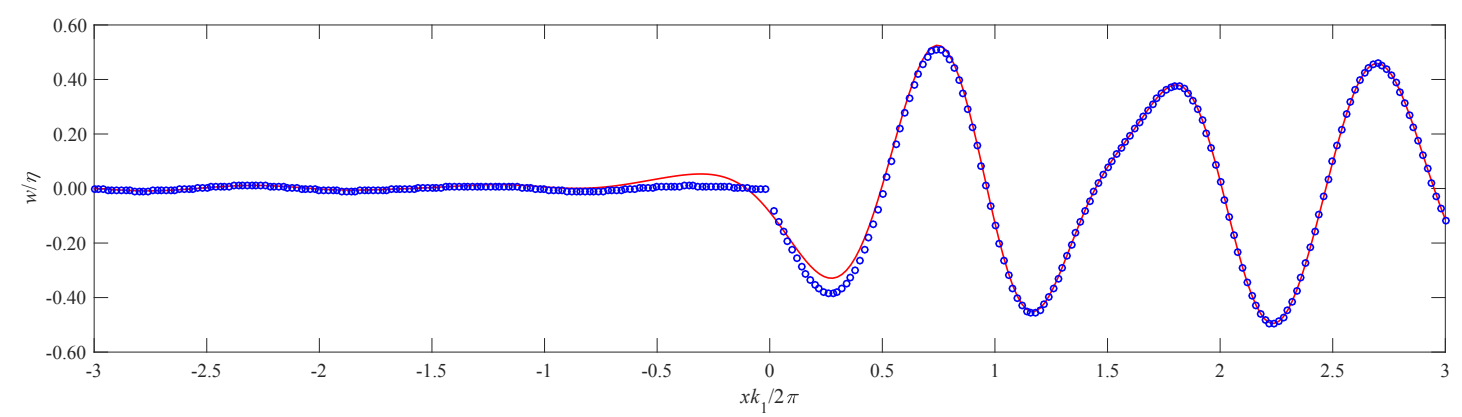

6

7

8

9

10

Fig. 28. Time history of the deformation at $x=0$ with different $\eta$ : solid line: fully linear solution; dashed line: $\eta / a=0.1$; dotted line: $\eta / a=0.4$; dash-dotted line: $\eta / a=0.8$. $\left(v a=0.5 ; h / a=2 ; h_{1} / a=0.15\right.$, $\left.D / a^{4}=15.368, K / a=0.135\right)$

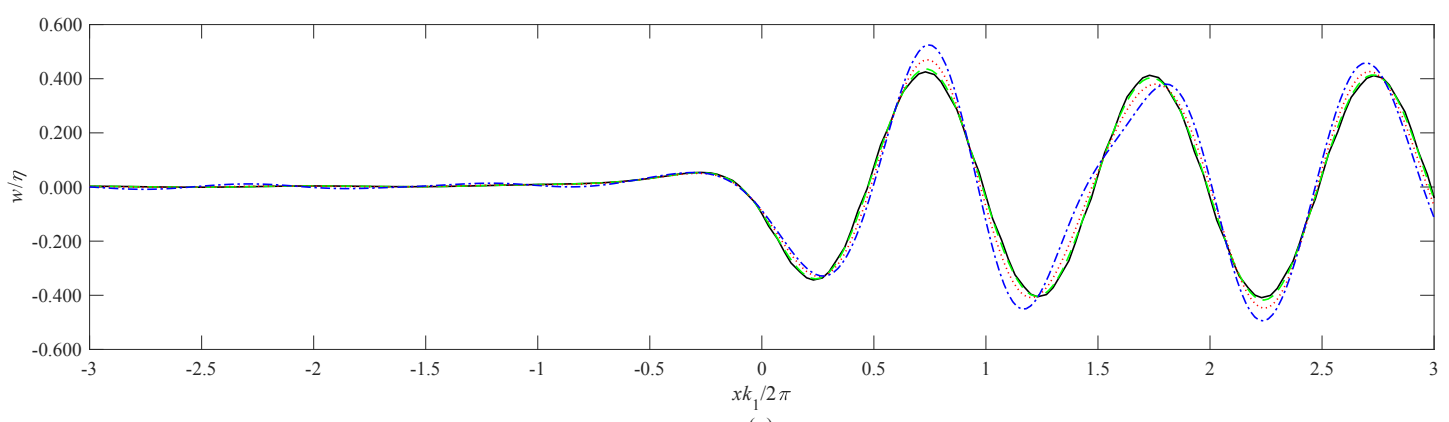

Fig. 29. Profile of the deformation at $t / T=0.5$. (a) profiles with different $\eta$ : solid line: fully linear solution; dashed line: $\eta / a=0.1$; dotted line: $\eta / a=0.4$; dash-dotted line: $\eta / a=0.8 ;(b)$ profiles at $\eta / a=0.8:$ solid line: Eq. (37); open circles: far field formula Eq. (38). ( $v a=0.5 ; h / a=2 ; h_{1} / a=0.15, D / a^{4}=15.368$, $K / a=0.135$

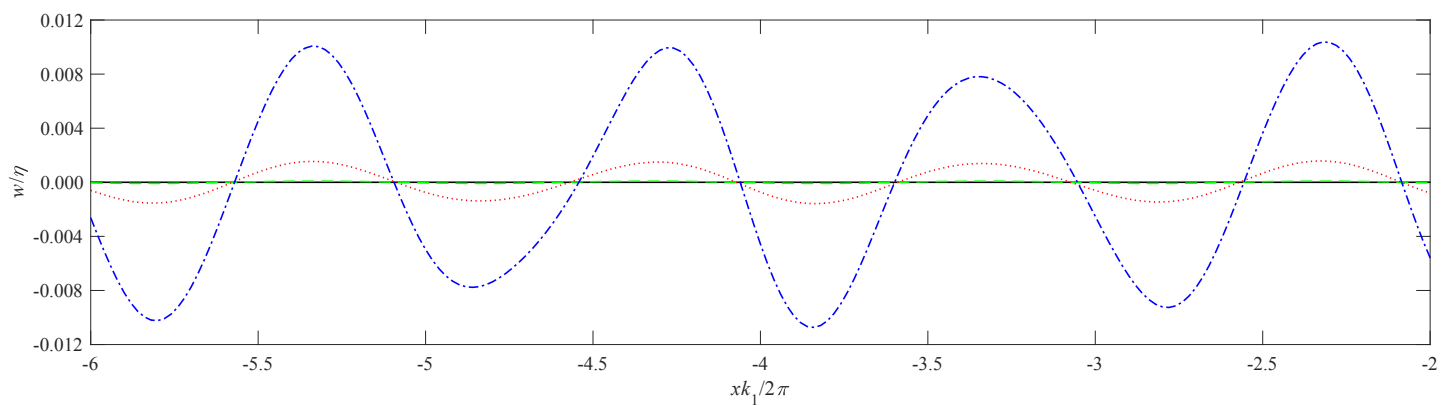

Fig. 30. The same as figure 29, but for the far field on the left hand side $(x<0)$ only calculated by the far field formula Eq. (38).

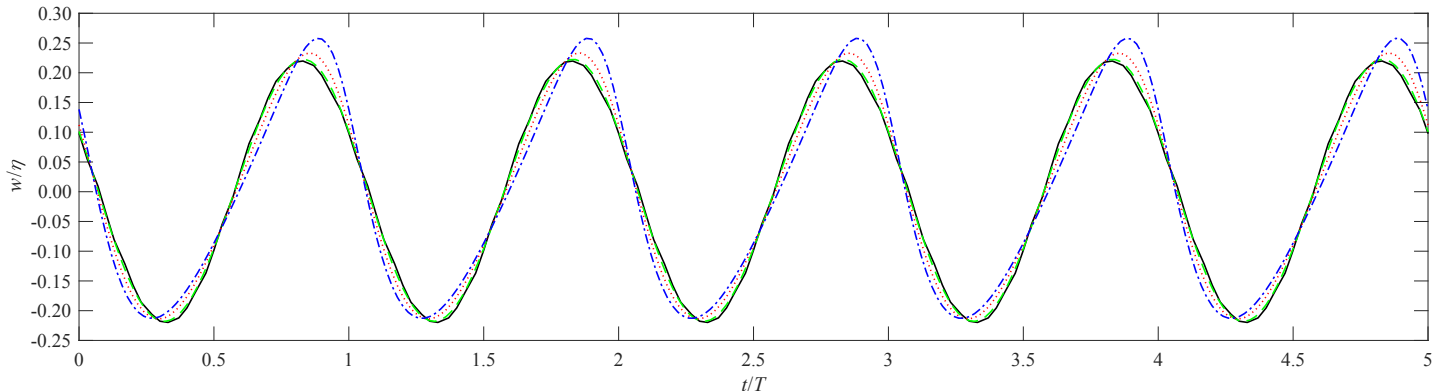


1 Simulations are then carried out for $v a=0.1,1,2$ and 4 respectively, with $\eta / a=0.8, h / a=2$ 2 and $h_{1} / a=0.15, D / a^{4}=15.368, K / a=0.135$. Fig. 31 and Fig. 32 respectively show the time histories of the horizontal force and vertical force. The behavior is very similar to that in the two previous cases. However, at each given $v$, the curve is far less regular. The corresponding time history and profile of the deformation of the ice sheet are presented in Fig. 33 and Fig. 34 respectively. It can be seen that the profile is not just dominated by the $k_{1}$ components, and other components from Eq. (15) are also important.

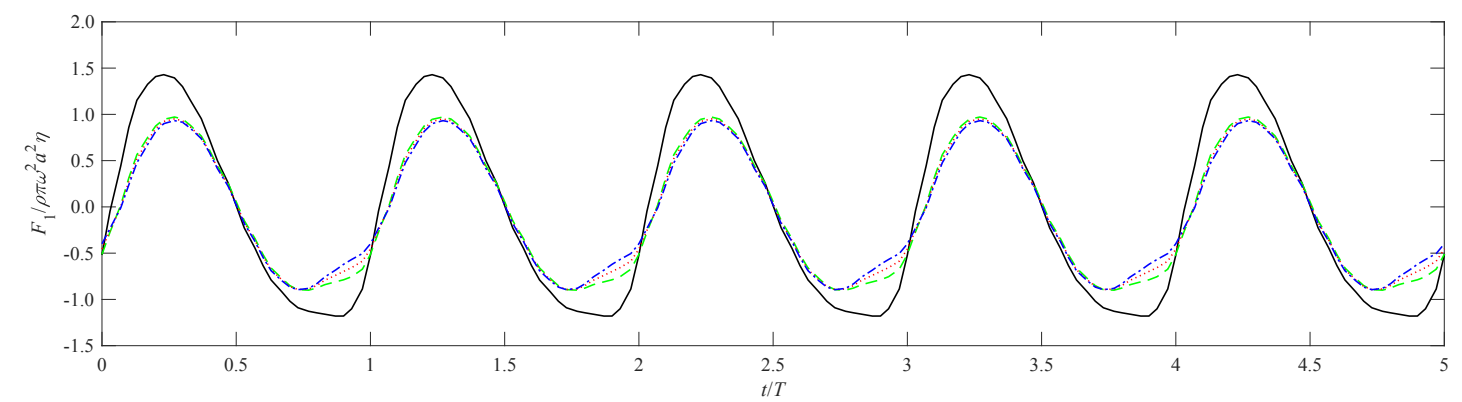

Fig. 31. Horizontal force at different $v$ : solid line: $v a=0.1$; dashed line: $v a=1$; dotted line: $v a=2$; dashdotted line: $v a=4 .\left(\eta / a=0.8 ; h / a=2 ; h_{1} / a=0.15, D / a^{4}=15.368, K / a=0.135\right)$

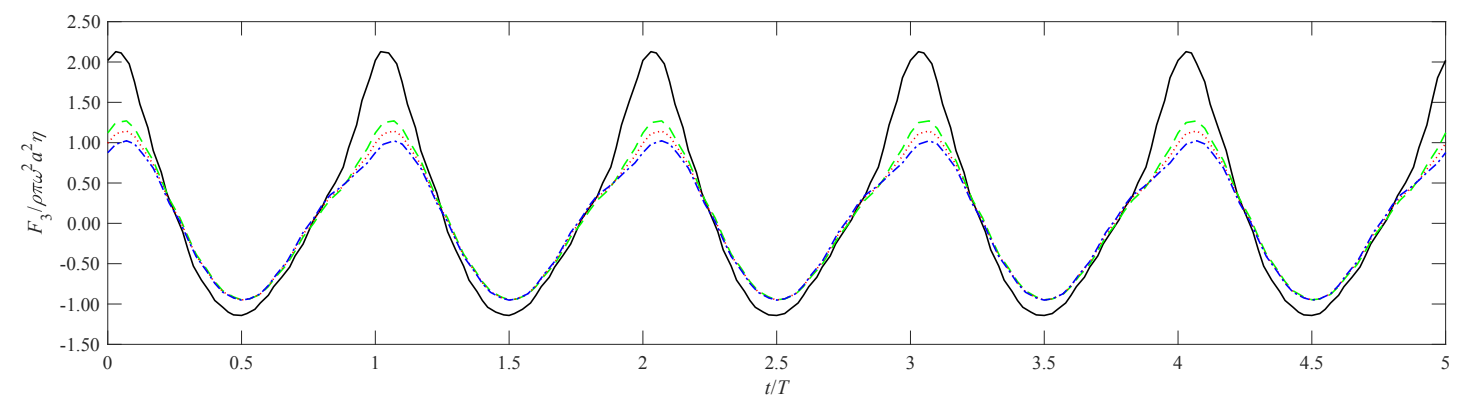

Fig. 32. Vertical force at different $v$ : solid line: $v a=0.1$; dashed line: $v a=1$; dotted line: $v a=2$; dashdotted line: $v a=4 .\left(\eta / a=0.8 ; h / a=2 ; h_{1} / a=0.15, D / a^{4}=15.368, K / a=0.135\right)$

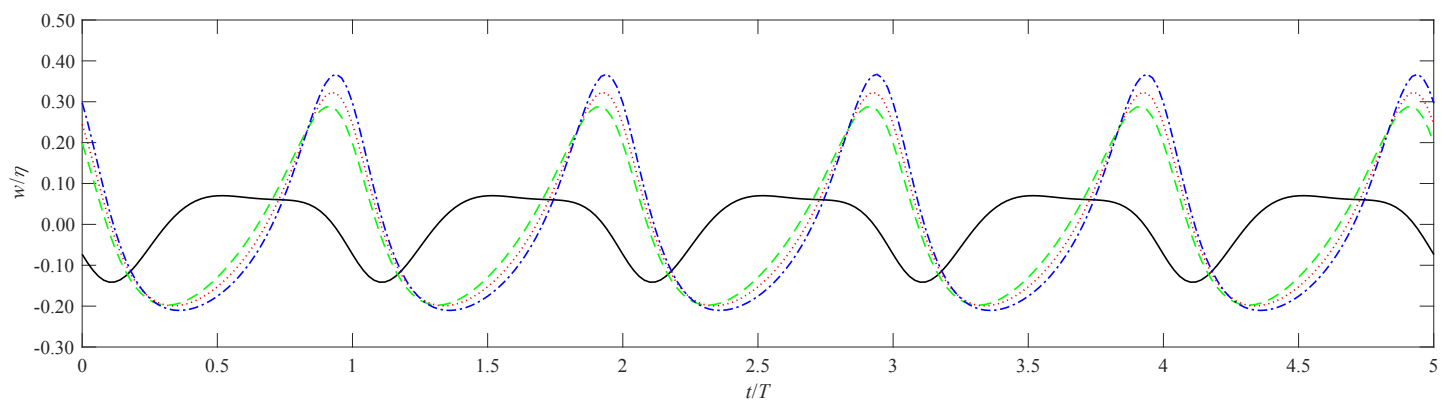

Fig. 33. Time history of the deformation at $x=0$ with different $v$ : solid line: $v a=0.1$; dashed line: $v a=1$; dotted line: $v a=2$; dash-dotted line: $v a=4 .\left(\eta / a=0.8 ; h / a=2 ; h_{1} / a=0.15, D / a^{4}=15.368\right.$, $K / a=0.135$ 
1

2

4

5

6

7

10

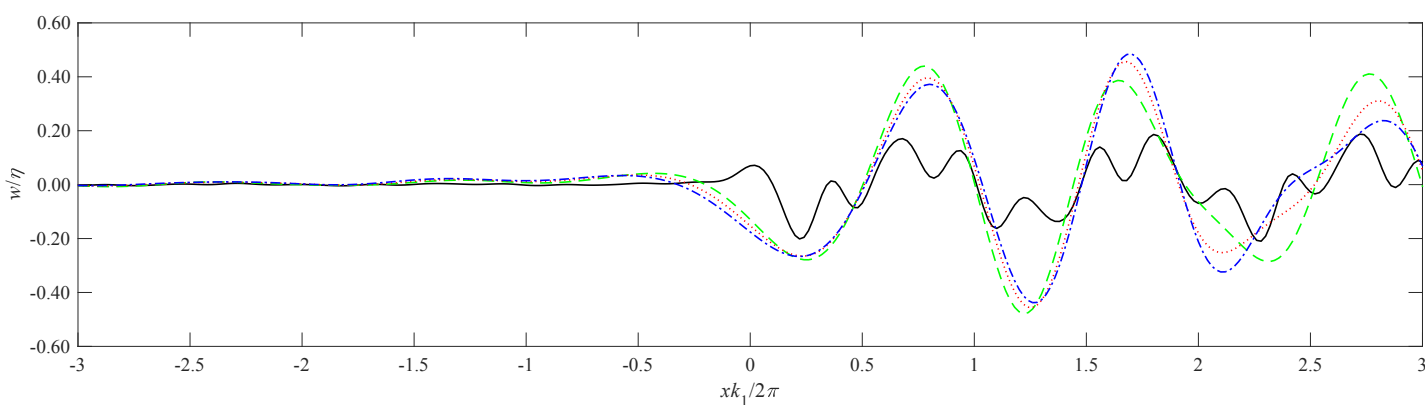

Fig. 34. Profile of the deformation at $t / T=0.5$ with different $v$ : solid line: $v a=0.1$; dashed line: $v a=1$; dotted line: $v a=2$; dash-dotted line: $v a=4 .\left(\eta / a=0.8 ; h / a=2 ; h_{1} / a=0.15, D / a^{4}=15.368\right.$, $K / a=0.135$

Forces for $h_{1} / a=0.05,0.1,0.15$ and 0.2 at $\eta_{1} / a=0.8, h / a=2$ and $v a=3$ are shown in Fig. 35 and Fig. 36. The figures show that the thickness has bigger effect on the force than that in the previous two cases. Higher oscillatory components are obvious for small $h_{1} / a$, partly because of the larger deformation of ice sheet in Figs. 37 and 38, as the ice sheet becomes more flexible.

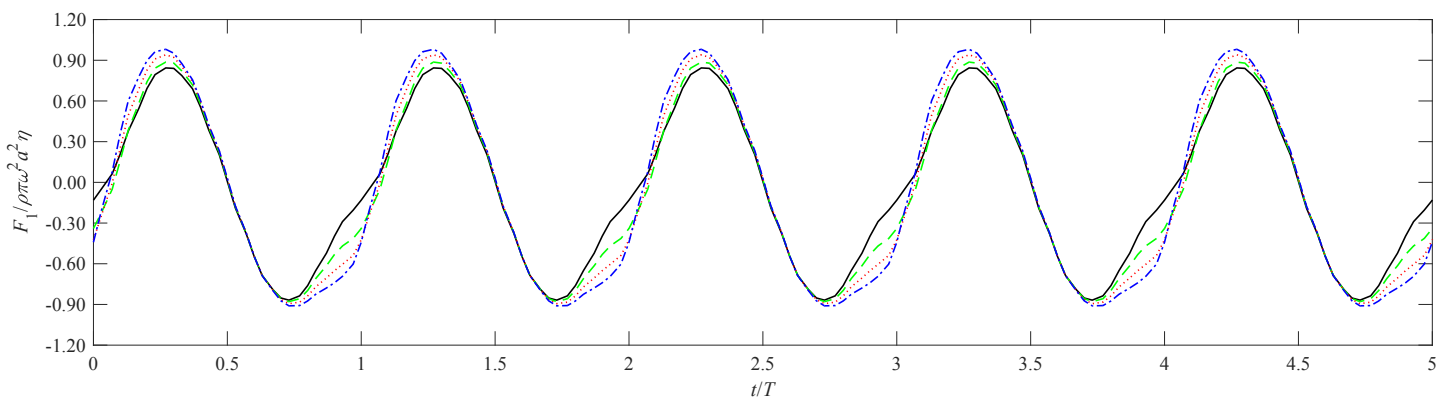

Fig. 35. Horizontal force at different $h_{1}$ : solid line: $h_{1} / a=0.05, D / a^{4}=0.569, K / a=0.045$; dashed line: $\quad h_{1} / a=0.1, \quad D / a^{4}=4.554, \quad K / a=0.09 ;$ dotted line: $h_{1} / a=0.15, D / a^{4}=15.368$, $K / a=0.135$; dash-dotted line: $h_{1} / a=0.2, D / a^{4}=36.429, K / a=0.18 .(\eta / a=0.8 ; v a=3$; $h / a=2)$

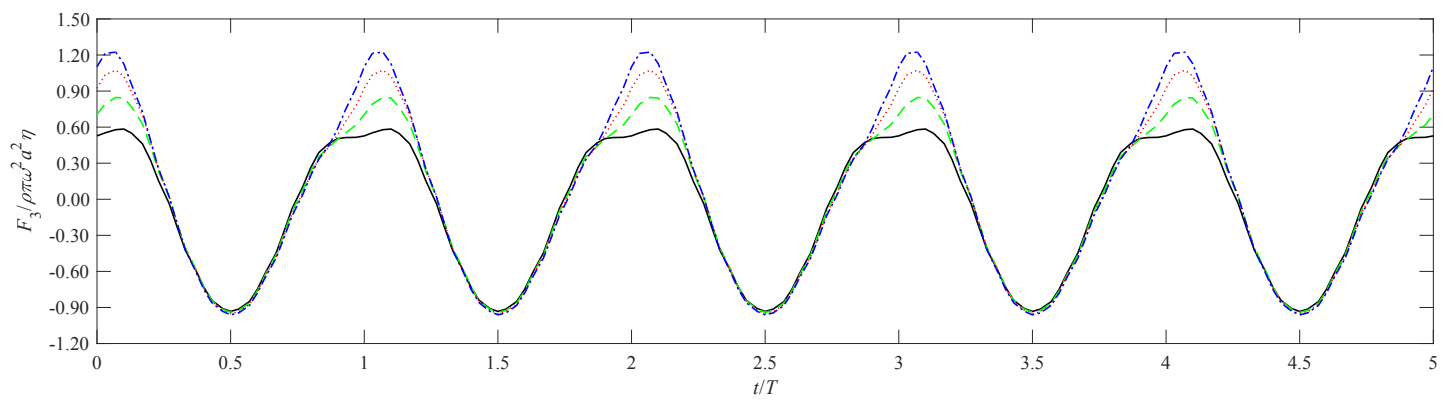

Fig. 36. Vertical force at different $h_{1}$ : solid line: $h_{1} / a=0.05, D / a^{4}=0.569, K / a=0.045$; dashed line: $h_{1} / a=0.1, D / a^{4}=4.554, K / a=0.09$; dotted line: $h_{1} / a=0.15, D / a^{4}=15.368, K / a=0.135$; dash-dotted line: $h_{1} / a=0.2, D / a^{4}=36.429, K / a=0.18 .(\eta / a=0.8 ; v a=3 ; h / a=2)$ 


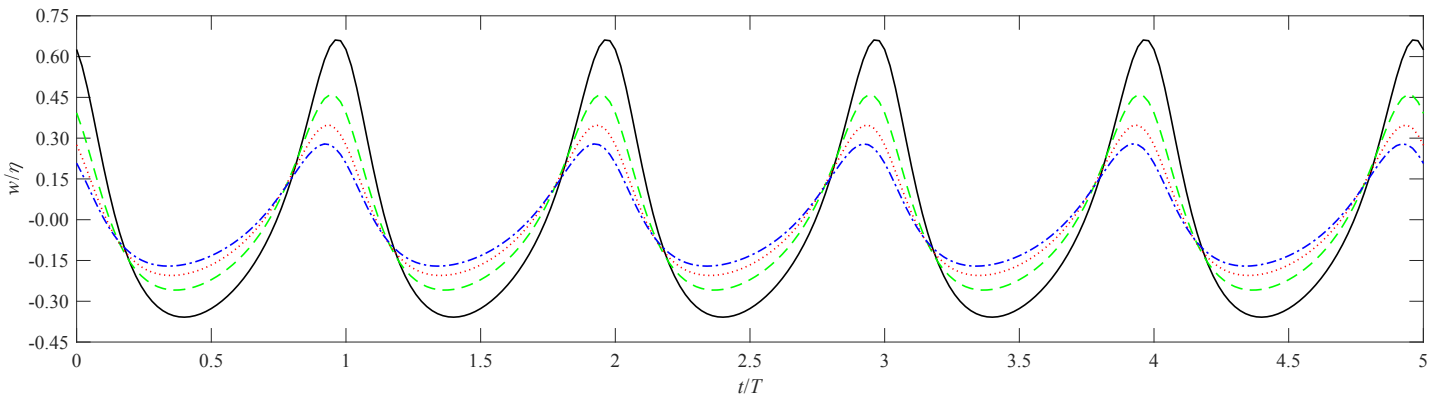

Fig. 37. Time history of the deformation at $x=0$ with different $h_{1}$ : solid line: $h_{1} / a=0.05, D / a^{4}=0.569$, $K / a=0.045$; dashed line: $h_{1} / a=0.1, D / a^{4}=4.554, K / a=0.09 ;$ dotted line: $h_{1} / a=0.15$, $D / a^{4}=15.368, K / a=0.135 ;$ dash-dotted line: $h_{1} / a=0.2, D / a^{4}=36.429, K / a=0.18$. $\eta / a=0.8 ; v a=3 ; h / a=2)$

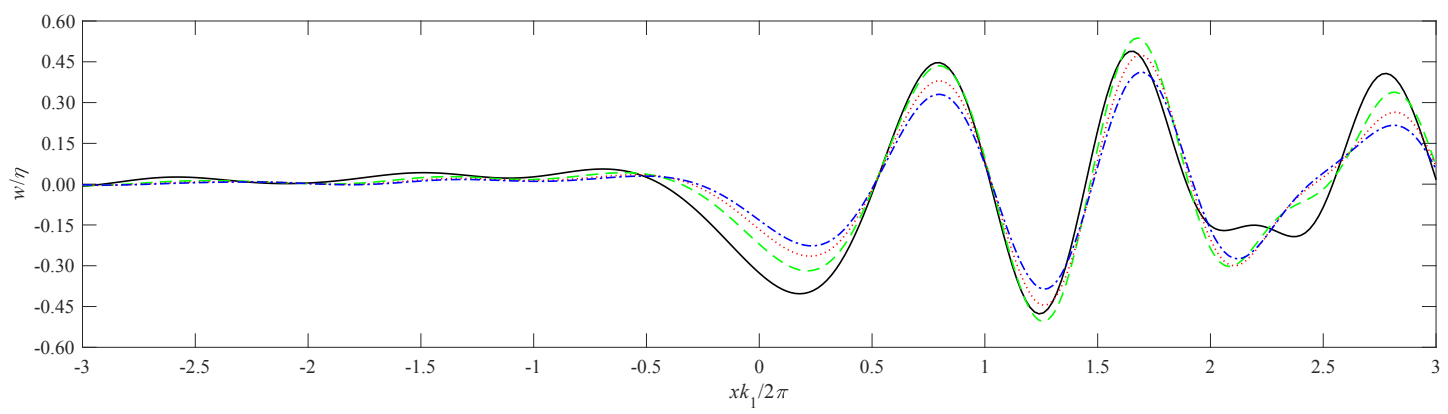

Fig. 38. Deformation of the ice sheet at $t / T=0.5$ with different $h_{1}:$ solid line: $h_{1} / a=0.05$, $D / a^{4}=0.569, K / a=0.045$; dashed line: $h_{1} / a=0.1, D / a^{4}=4.554, K / a=0.09$; dotted line: $h_{1} / a=0.15, D / a^{4}=15.368, K / a=0.135 ;$ dash-dotted line: $h_{1} / a=0.2, D / a^{4}=36.429$, $K / a=0.18 .(\eta / a=0.8 ; v a=3 ; h / a=2)$

\section{Conclusions}

The multipole expansion method has been used to investigate the problem of a submerged circular cylinder undergoing large amplitude oscillations in water covered by an ice sheet. The method and solution procedure are verified by the convergence study and comparisons. Simulations are then carried out for various cases, from which the following conclusions can be drawn:

(1) For the purely horizontal motion, the problem is anti-symmetric when the problem is fully linearized. When fully nonlinear condition is imposed on the body surface, the problem becomes neither symmetric nor anti-symmetric. The motion contains components with frequencies of $n \omega$, $n=1,2, \ldots$, instead of $n=1$ as in the linear case. In the ice sheet deformation, the components are symmetric when $n$ is an even number and anti-symmetric when $n$ is an odd number. The horizontal force will have components of $(2 n+1) \omega$ only and vertical force of $2 n \omega$ only. The higher oscillatory components become more obvious when the motion amplitude $\eta_{1}$ increases.

(2) The nonlinearity effect becomes more obvious in vertical motion as the distance from the body centre to the ice sheet changes during the oscillation. 
1 (3) For clockwise circular motion, when the body surface boundary condition is linearized there is 2 no wave at $x=-\infty$. When fully nonlinear boundary condition is imposed on the body surface, the 3 solution shows that there are waves at $x=-\infty$, although they may be still relatively small. The effect of nonlinearity in the circular motion is more complex than that in the horizontal or vertical motion.

(4) With some typical physical properties of the ice sheet, the solution of the dispersion relationship shows that the wave number is far less sensitive to the oscillation frequency than that in the free surface problem. As a result, as the oscillation frequency increases, its effect on the results becomes far less obvious.

(5) At a given frequency when the ice thickness $h_{1}$ increases, both the horizontal and vertical forces become larger, while the deformation of the ice sheet becomes smaller due to the fact that the ice sheet becomes more rigid.

The present work has also provided some accurate and reliable results for verifying numerical methods for more general problems. This is particularly important here as the boundary condition on the ice sheet involves the fifth derivative. When a Rankine source method is used for such a problem with ice sheet of non uniform physical properties, the calculation of this high derivative will be a major challenge. This challenge is already fully recognized by the well known second derivative of the m-term [23] in the wave/current/body problem and some special treatments are needed (e.g. Wu [24]). The present work therefore provides a useful way to verify this kind of numerical method.

\section{Acknowledgements}

This work is supported by Lloyd's Register Foundation through the joint centre involving University College London, Shanghai Jiaotong University and Harbin Engineering University, to which the authors are most grateful. Lloyd's Register Foundation helps to protect life and property by supporting engineering-related education, public engagement, and the application of research. This work is also supported by the National Natural Science Foundation of China (Grant No. 11472088)

\section{Appendix. Far field formula for horizontal steady force}

There are a large number of equations for the linear free surface flow problem, from which some of the forces on the body can be obtained from the wave radiated into infinity [25]. Far field equation was also derived by $\mathrm{Wu}[18]$ for the steady force on the body undergoing large amplitude motion. For the present case, the derivation may be less straightforward because of the complexity in the upper surface boundary condition.

The horizontal force in Eq. (29) can be given as [18] 


$$
\begin{aligned}
F_{1} & =-\rho \frac{d}{d t} \int_{S_{0}} \Phi n_{1} \mathrm{~d} s+\frac{\rho}{2} \int_{S_{0}}\left(\frac{\partial \Phi}{\partial x} \frac{\partial \Phi}{\partial n}-\frac{\partial^{2} \Phi}{\partial n \partial x} \Phi\right) \mathrm{d} s \\
& =-\rho \frac{d}{d t} \int_{S_{0}} \Phi n_{1} \mathrm{~d} s-\frac{\rho}{2} \int_{S_{F}}\left(\frac{\partial \Phi}{\partial x} \frac{\partial \Phi}{\partial n}-\frac{\partial^{2} \Phi}{\partial n \partial x} \Phi\right) \mathrm{d} s-\frac{\rho}{2} \int_{S_{\infty}}\left(\frac{\partial \Phi}{\partial x} \frac{\partial \Phi}{\partial n}-\frac{\partial^{2} \Phi}{\partial n \partial x} \Phi\right) \mathrm{d} s
\end{aligned}
$$

2 where $S_{\infty}$ comprises two vertical lines at $x= \pm \infty$ respectively.

3 The steady component can be obtained from the average over the period $T=2 \pi / \omega$, or

$$
F_{1}(0)=\frac{1}{T} \int_{0}^{T} F_{1} \mathrm{~d} t=F_{1, \infty}(0)+F_{1, F}(0)
$$

5 where

6

7 and

$$
F_{1, \infty}(0)=-\frac{\rho}{2 T} \int_{0}^{T} \int_{S_{\infty}}\left(\frac{\partial \Phi}{\partial x} \frac{\partial \Phi}{\partial n}-\frac{\partial^{2} \Phi}{\partial n \partial x} \Phi\right) \mathrm{d} s \mathrm{~d} t
$$

$$
F_{1, F}(0)=-\frac{\rho}{2 T} \int_{0}^{T} \int_{S_{F}}\left(\frac{\partial \Phi}{\partial x} \frac{\partial \Phi}{\partial n}-\frac{\partial^{2} \Phi}{\partial n \partial x} \Phi\right) \mathrm{d} s \mathrm{~d} t
$$

Let $x \rightarrow \pm \infty$ in Eq. (14) and using Eq. (13.16) of Wehausen and Laitone [26], we obtain the asymptotic expressions for the velocity potentials as

$$
\begin{aligned}
& \lim _{x \rightarrow+\infty} \Phi=\operatorname{Re}\left\{\sum_{s=1}^{\infty}\left[f_{1}(s) \mathrm{e}^{\mathrm{i} \lambda x} \mathrm{e}^{\lambda z} \mathrm{e}^{-\mathrm{i} s \omega t}+f_{2}(s) \mathrm{e}^{-\mathrm{i} \lambda x} \mathrm{e}^{\lambda z} \mathrm{e}^{\mathrm{i} s \omega t}\right]\right\} \\
& \lim _{x \rightarrow-\infty} \Phi=\operatorname{Re}\left\{\sum_{s=1}^{\infty}\left[g_{1}(s) \mathrm{e}^{\mathrm{i} \lambda x} \mathrm{e}^{\lambda z} \mathrm{e}^{\mathrm{i} s \omega t}+g_{2}(s) \mathrm{e}^{-\mathrm{i} \lambda x} \mathrm{e}^{\lambda z} \mathrm{e}^{-\mathrm{i} s \omega t}\right]\right\}
\end{aligned}
$$

where

$$
f_{1}(s)=\sum_{m=1}^{\infty} \sum_{p=-\infty}^{\infty} \sum_{q=-\infty}^{\infty} C_{m}^{-s-p-q} \frac{a^{m}}{(m-1) !}(-\mathrm{i})^{q} \mathrm{e}^{\mathrm{i} p \gamma_{3}+\mathrm{i} q \gamma_{1}}\left[2 \mathrm{i} \pi \frac{\mathrm{e}^{-\lambda h} \lambda^{m-1} I_{p}\left(\lambda \eta_{3}\right) J_{q}\left(\lambda \eta_{1}\right) \alpha(\lambda,-s)}{\beta^{\prime}(\lambda,-s)}\right]
$$

$$
\begin{aligned}
& f_{2}(s)=\sum_{m=1}^{\infty} \sum_{p=-\infty}^{\infty} \sum_{q=-\infty}^{\infty} D_{m}^{s-p-q} \frac{a^{m}}{(m-1) !}(+\mathrm{i})^{q} \mathrm{e}^{\mathrm{i} p \gamma_{3}+\mathrm{i} q \gamma_{1}}\left[(-2) \mathrm{i} \pi \frac{\mathrm{e}^{-\lambda h} \lambda^{m-1} I_{p}\left(\lambda \eta_{3}\right) J_{q}\left(\lambda \eta_{1}\right) \alpha(\lambda, s)}{\beta^{\prime}(\lambda, s)}\right] \\
& g_{1}(s)=\sum_{m=1}^{\infty} \sum_{p=-\infty}^{\infty} \sum_{q=-\infty}^{\infty} C_{m}^{s-p-q} \frac{a^{m}}{(m-1) !}(-\mathrm{i})^{q} \mathrm{e}^{\mathrm{i} p \gamma_{3}+\mathrm{i} q \gamma_{1}}\left[-2 \mathrm{i} \pi \frac{\mathrm{e}^{-\lambda h} \lambda^{m-1} I_{p}\left(\lambda \eta_{3}\right) J_{q}\left(\lambda \eta_{1}\right) \alpha(\lambda, s)}{\beta^{\prime}(\lambda, s)}\right]
\end{aligned}
$$

$$
\begin{gathered}
g_{2}(s)=\sum_{m=1}^{\infty} \sum_{p=-\infty}^{\infty} \sum_{q=-\infty}^{\infty} D_{m}^{-s-p-q} \frac{a^{m}}{(m-1) !}(+\mathrm{i})^{q} \mathrm{e}^{\mathrm{i} p \gamma_{3}+\mathrm{i} q \gamma_{1}}\left[2 \mathrm{i} \pi \frac{\mathrm{e}^{-\lambda h} \lambda^{m-1} I_{p}\left(\lambda \eta_{3}\right) J_{q}\left(\lambda \eta_{1}\right) \alpha(\lambda,-s)}{\beta^{\prime}(\lambda,-s)}\right] \\
\alpha(k, s)=k\left(D k^{4}-K v s^{2}+1\right)+v s^{2} \\
\beta(k, s)=k\left(D k^{4}-K v s^{2}+1\right)-v s^{2}
\end{gathered}
$$

The integration over $S_{\infty}$ can be rewritten as

$$
\begin{aligned}
F_{1, \infty}(0)= & -\left.\frac{\rho}{2 T} \int_{0}^{T} \int_{-\infty}^{0}\left(\frac{\partial \Phi}{\partial x} \frac{\partial \Phi}{\partial x}-\frac{\partial^{2} \Phi}{\partial x^{2}} \Phi\right)\right|_{x \rightarrow+\infty} \mathrm{d} z \mathrm{~d} t \\
& +\left.\frac{\rho}{2 T} \int_{0}^{T} \int_{-\infty}^{0}\left(\frac{\partial \Phi}{\partial x} \frac{\partial \Phi}{\partial x}-\frac{\partial^{2} \Phi}{\partial x^{2}} \Phi\right)\right|_{x \rightarrow-\infty} \mathrm{d} z \mathrm{~d} t
\end{aligned}
$$

Substituting Eqs. (64) and (65) into the above equation, we have

$$
F_{1, \infty}(0)=-\frac{\rho}{4} \sum_{s=1}^{\infty} \lambda\left(|f(s)|^{2}-|g(s)|^{2}\right)
$$

where 


$$
\begin{aligned}
& f(s)=\bar{f}_{1}(s)+f_{2}(s) \\
& g(s)=\bar{g}_{1}(s)+g_{2}(s)
\end{aligned}
$$

To compute the integral over $S_{F}$ in Eq. (63), we notice Eqs. (7) and (10) and write the potential as

$$
\Phi=\operatorname{Re}\left(\sum_{s=-\infty}^{+\infty} \phi_{s}^{\prime} \mathrm{e}^{\mathrm{i} s \omega t}\right)
$$

Invoking Eqs. (64) and (65), we have

$$
\begin{aligned}
& \left.\phi_{s}^{\prime}\right|_{+\infty}=\left[\delta(s+|s|) f_{1}(-s) \mathrm{e}^{\mathrm{i} \lambda x}+\delta(s-|s|) f_{2}(s) \mathrm{e}^{-\mathrm{i} \lambda x}\right] \mathrm{e}^{\lambda z} \\
& \left.\phi_{s}^{\prime}\right|_{-\infty}=\left[\delta(s-|s|) g_{1}(s) \mathrm{e}^{\mathrm{i} \lambda x}+\delta(s+|s|) g_{2}(-s) \mathrm{e}^{-\mathrm{i} \lambda x}\right] \mathrm{e}^{\lambda z}
\end{aligned}
$$

where the function $\delta(s)$ has been defined below Eq. (19). Eq. (72) can be also written as

$$
\Phi=\sum_{s=-\infty}^{+\infty} \phi_{s} \mathrm{e}^{\mathrm{i} s \omega t}
$$

where

$$
\phi_{s}=\frac{1}{2}\left(\phi_{s}^{\prime}+\bar{\phi}_{-s}^{\prime}\right)
$$

From Eqs. (73), (74) and (76), we have

$$
\begin{aligned}
\left.\phi_{s}\right|_{+\infty}= & \frac{1}{2}\left\{\left[\delta(s+|s|) f_{1}(-s) \mathrm{e}^{\mathrm{i} \lambda x}+\delta(s-|s|) f_{2}(s) \mathrm{e}^{-\mathrm{i} \lambda x}\right]\right. \\
& \left.+\left[\delta(-s+|-s|) \bar{f}_{1}(s) \mathrm{e}^{-\mathrm{i} \lambda x}+\delta(-s-|-s|) \bar{f}_{2}(-s) \mathrm{e}^{\mathrm{i} \lambda x}\right]\right\} \mathrm{e}^{\lambda z} \\
\left.\phi_{s}\right|_{-\infty}= & \frac{1}{2}\left\{\left[\delta(s-|s|) g_{1}(s) \mathrm{e}^{\mathrm{i} \lambda x}+\delta(s+|s|) g_{2}(-s) \mathrm{e}^{-\mathrm{i} \lambda x}\right]\right. \\
+ & {\left.\left[\delta(-s-|-s|) \bar{g}_{1}(-s) \mathrm{e}^{-\mathrm{i} \lambda x}+\delta(-s+|-s|) \bar{g}_{2}(s) \mathrm{e}^{\mathrm{i} \lambda x}\right]\right\} \mathrm{e}^{\lambda z} }
\end{aligned}
$$

Substitution of Eq. (75) into Eq. (5) yields

$$
\begin{aligned}
\phi_{s} & =\mathrm{g} D /(p \omega)^{2} \phi_{s, z x x x}+\left(g /(s \omega)^{2}-K\right) \phi_{s, z} \\
\phi_{s, x} & =\mathrm{g} D /(p \omega)^{2} \phi_{s, z x x x x x}+\left(g /(s \omega)^{2}-K\right) \phi_{s, z x}
\end{aligned}
$$

Substituting Eq. (75) into Eq. (63) and using the above two equations, we obtain

$$
\begin{aligned}
F_{1, F}(0) & =-\frac{\rho}{2 T} \int_{0}^{T} \mathrm{~d} t \int_{S_{F}}\left(\frac{\partial \Phi}{\partial x} \frac{\partial \Phi}{\partial n}-\frac{\partial^{2} \Phi}{\partial n \partial x} \Phi\right) \mathrm{d} s \\
& =-\frac{\rho}{2 T} \int_{0}^{T} \mathrm{~d} t \sum_{s=-\infty}^{+\infty} \sum_{s_{1}=-\infty}^{+\infty} \mathrm{e}^{\mathrm{i}\left(s+s_{1}\right) \omega t} \int_{S_{F}}\left[\left(\mathrm{~g} D /(s \omega)^{2}\right)\left(\phi_{s, z x x x x} \phi_{s_{1}, z}-\phi_{s, z x x x x} \phi_{s_{1}, z x}\right)\right] \mathrm{d} s \\
& -\frac{\rho}{2 T} \int_{0}^{T} \mathrm{~d} t \sum_{s=-\infty}^{+\infty} \sum_{S_{1}=-\infty}^{+\infty} \mathrm{e}^{\mathrm{i}\left(s+s_{1}\right) \omega t} \int_{S_{F}}\left[\left(g /(s \omega)^{2}\right)\left(\phi_{s, z x} \phi_{s_{1}, z}-\phi_{s, z} \phi_{s_{1}, z x}\right)\right] \mathrm{d} s \\
& +\frac{\rho}{2 T} \int_{0}^{T} \mathrm{~d} t \sum_{s=-\infty}^{+\infty} \sum_{s_{1}=-\infty}^{+\infty} \mathrm{e}^{\mathrm{i}\left(s+s_{1}\right) \omega t} \int_{S_{F}}\left[K\left(\phi_{s, z x} \phi_{s_{1}, z}-\phi_{s, z} \phi_{s_{1}, z x}\right)\right] \mathrm{d} s
\end{aligned}
$$

In each integration over $S_{F}, s$ and $s_{1}$ may be exchanged in the second term. Noticing the fact that only the terms $s+s_{1}=0$ have contributions to the steady force, we can conclude that only the first integration is non zero. Through integration by parts, we have

$$
F_{1, F}(0)=-\frac{\rho}{2} \sum_{s=-\infty}^{+\infty}\left[\mathrm{g} D /(s \omega)^{2}\right]
$$

$$
\times\left.\left[\left(\phi_{s, z x x x} \phi_{-s, z}-\phi_{s, z x x x} \phi_{-s, z x}\right)-\left(\phi_{s, z x x} \phi_{-s, z x}-\phi_{s, z x x} \phi_{-s, z x x}\right)\right]\right|_{-\infty} ^{+\infty}
$$

Invoking Eq. (76), we have 


$$
\phi_{-s}=\bar{\phi}_{s}
$$

2 Thus Eq. (82) can be rewritten as

3

$$
\begin{aligned}
F_{1, F}(0)=-\rho \operatorname{Re} & \left\{\sum_{s=1}^{+\infty}\left[\mathrm{g} D /(s \omega)^{2}\right]\right. \\
& \left.\times\left.\left(\phi_{s, z x x x x} \bar{\phi}_{s, z}-2 \phi_{s, z x x x} \bar{\phi}_{s, z x}+\phi_{s, z x x} \bar{\phi}_{s, z x x}\right)\right|_{-\infty} ^{+\infty}\right\}
\end{aligned}
$$

Substituting Eqs. (77) and (78) into Eq. (84), we obtain

$$
F_{1, F}(0)=-\rho \sum_{s=1}^{+\infty}\left[\mathrm{g} D /(s \omega)^{2}\right] \lambda^{6}\left(|f(s)|^{2}-|g(s)|^{2}\right)
$$

Finally, we have

$$
F_{1}(0)=-\rho \sum_{s=1}^{+\infty}\left[\frac{\mathrm{g} D}{(s \omega)^{2}} \lambda^{6}+\frac{1}{4} \lambda\right]\left[|f(s)|^{2}-|g(s)|^{2}\right]
$$

\section{References}

[1] V.A. Squire, J.P. Dugan, P. Wadhams, a. P J Rottier, A.K. Liu, Of ocean waves and sea ice, Annual Review of Fluid Mechanics, 27 (1995) 115-168.

[2] V.A. Squire, Past, present and impendent hydroelastic challenges in the polar and subpolar seas, Philosophical Transactions, 369 (2011) 2813-2831.

[3] C. Fox, V.A. Squire, Reflection and transmission characteristics at the edge of shore fast sea ice, Journal of Geophysical Research Oceans, 95 (1990) 11629-11639.

[4] C. Fox, V.A. Squire, On the Oblique Reflexion and Transmission of Ocean Waves at Shore Fast Sea Ice, Philosophical Transactions of the Royal Society A Mathematical Physical \& Engineering Sciences, 347 (1994) 185-218.

[5] T. Sahoo, T.L. Yip, A.T. Chwang, Scattering of surface waves by a semi-infinite floating elastic plate, Physics of Fluids, 13 (2001) 3215-3222.

[6] R. Eatock Taylor, M. Ohkusu, Green functions for hydroelastic analysis of vibrating free-free beams and plates, Applied Ocean Research, 22 (2000) 295-314.

[7] N.J. Balmforth, R.V. Craster, Ocean waves and ice sheets, Journal of Fluid Mechanics, 395 (1999) 89-124.

[8] L.A. Tkacheva, Hydroelastic behavior of a floating plate in waves, Journal of Applied Mechanics \& Technical Physics, 42 (2001) 991-996.

[9] L.A. Tkacheva, The diffraction of surface waves by a floating elastic plate at oblique incidence, Journal of Applied Mathematics \& Mechanics, 68 (2004) 425-436.

[10] H. Chung, C. Fox, Calculation of wave-ice interaction using the wiener-hopf technique, New Zealand J Math, 31 (2002) 1-18.

[11] I.V. Sturova, A.A. Korobkin, Two-dimensional problem of periodic loading of an elastic plate floating on the surface of an infinitely deep fluid, Journal of Applied Mechanics \& Technical Physics, 46 (2005) 355-364. 
1 [12] D. Das, B.N. Mandal, Oblique wave scattering by a circular cylinder submerged beneath an 2 ice-cover, International Journal of Engineering Science, 44 (2006) 166-179.

3 [13] I.V. Sturova, Wave generation by an oscillating submerged cylinder in the presence of a 4 floating semi-infinite elastic plate, Fluid Dynamics, 49 (2014) 504-514.

5 [14] I.V. Sturova, The effect of a crack in an ice sheet on the hydrodynamic characteristics of a 6 submerged oscillating cylinder, Journal of Applied Mathematics \& Mechanics, 79 (2015) 170-178.

7 [15] I.V. Sturova, Radiation of waves by a cylinder submerged in water with ice floe or polynya, 8 Journal of Fluid Mechanics, 784 (2015) 373-395.

9 [16] A.A. Savin, A.S. Savin, Ice cover perturbation by a dipole in motion within a liquid, Fluid Dynamics, 47 (2012) 139-146.

[17] A.T. Il'ichev, A.A. Savin, A.S. Savin, Formation of a wave on an ice-sheet above the dipole, moving in a fluid, Doklady Physics, 57 (2012) 202-205.

[18] G.X. Wu, Hydrodynamic forces on a submerged circular cylinder undergoing large-amplitude motion, Journal of Fluid Mechanics, 254 (1993) 41-58.

[19] F. Ursell, On the heaving motion of a circular cylinder on the surface of a fluid, Quarterly Journal of Mechanics \& Applied Mathematics, 2 (1949) 215-231.

[20] G.X. Wu, A note on non-linear hydrodynamic forces on a body submerged below a free surface, Applied Ocean Research, 15 (1994) 371-372.

[21] G.X. Wu, A note on non-linear hydrodynamic force on a floating body, Applied Ocean Research, 22 (2000) 315-316.

[22] T.F. Ogilvie, First- and second-order forces on a cylinder submerged under a free surface, Journal of Fluid Mechanics, 16 (1963) 451-472.

[23] J.N. Newman, The Theory of Ship Motions, Advances in Applied Mechanics, 18 (1979) 221283.

[24] G.X. Wu, A numerical scheme for calculating the mj terms in wave-current-body interaction problem, Applied Ocean Research, 13 (1991) 317-319.

[25] C.C. Mei, M. Stiassnie, K.P. Yue, Theory and Applications of Ocean Surface Waves Part 1: Linear Aspects, World Scientific Publishing Co.pte.ltd.hackensack Nj, (2005).

[26] J.V. Wehausen, E.V. Laitone, Surface Waves, In: Handbach des Physik, Springer, Berlin Verlag, (1960), 446-778. 